\title{
GLOBAL OPTIMIZATION WITH ORTHOGONALITY CONSTRAINTS VIA STOCHASTIC DIFFUSION ON MANIFOLD
}

\author{
HONGLIN YUAN*, XIAOYI GU*, RONGJIE LAI ${ }^{\dagger}$, AND ZAIWEN WEN
}

\begin{abstract}
Orthogonality constrained optimization is widely used in applications from science and engineering. Due to the nonconvex orthogonality constraints, many numerical algorithms often can hardly achieve the global optimality. We aim at establishing an efficient scheme for finding global minimizers under one or more orthogonality constraints. The main concept is based on noisy gradient flow constructed from stochastic differential equations (SDE) on the Stiefel manifold, the differential geometric characterization of orthogonality constraints. We derive an explicit representation of SDE on the Stiefel manifold endowed with a canonical metric and propose a numerically efficient scheme to simulate this SDE based on Cayley transformation with theoretical convergence guarantee. The convergence to global optimizers is proved under second-order continuity. The effectiveness and efficiency of the proposed algorithms are demonstrated on a variety of problems including homogeneous polynomial optimization, computation of stability number, and 3D structure determination from Common Lines in Cryo-EM.
\end{abstract}

Key words. Orthogonality constrained optimization, Global optimization, Stochastic differential equations, Stochastic diffusion on manifold

AMS subject classifications. 90C26, 65K05, 49Q99

1. Introduction. Mathematically, the orthogonality constrained problem can be formulated as the following form:

$$
\min _{X \in \mathbb{R}^{n \times p}} \mathcal{F}(X), \quad \text { s.t. } X^{\top} X=I_{p}
$$

where $\mathcal{F}$ is a smooth objective function and $I_{p}$ indicates the $p$-by- $p$ identity matrix. The feasible set $\mathcal{M}_{n, p}=\left\{X \in \mathbb{R}^{n \times p}: X^{\top} X=I_{p}\right\}$ is well-known as the Stiefel manifold (once equipped with its natural submanifold structure from $\mathbb{R}^{n \times p}$ ). We also denote it by $\mathcal{M}$ if there is no ambiguity on the dimensions.

Particularly in the case of $p=1$, the above problem is known as the spherically constrained problem. In the case of $p=n$, the feasible set becomes orthogonal group $\mathcal{O}_{n}$, where the feasible matrices are square and orthogonal. More generally, the following optimization problem with multiple orthogonality (or spherical) constraints is widely used in many problems such as conformal mapping [12, 15], p-Harmonic flow [17, 32, 33, 11], 1-bit compressive sensing [4, 16], compressed modes [26], the graph stability number, Cryo-electron microscopy (Cryo-EM) [31], nonlinear eigenvalue problem in density functional theory $[19,35]$ as well as dictionary learning $[2,5]$ :

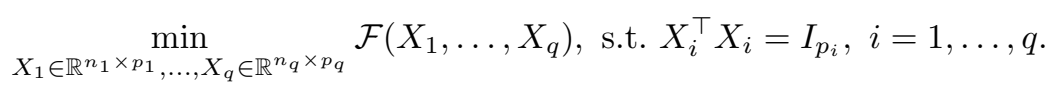

Non-convexity is one of the major challenges of problems (1) and (2) since there might be mulitiple local minimizers, from which finding global minimizers is generally NP-hard. Most existing algorithms [1, 36, 23] on the Stiefel manifold focus on finding local optimizers without exploiting the global structures, and thus there is no guarantee to obtain the global minimizers except for some trivial cases.

\footnotetext{
*Computational Mathematics, Peking University, China (yhlmath@pku.edu.cn, xiaoyigu@pku.edu.cn).

${ }^{\dagger}$ Department of Mathematics, Rensselaer Polytechnic Institute, Troy, NY 12180 (lair@rpi.edu).

${ }^{\ddagger}$ Beijing International Center for Mathematical Research, Peking University, China (wenzw@pku.edu.cn).
} 
1.1. Local feasible solver on Stiefel manifold. One step of our algorithm is mostly based on the first-order algorithms proposed in [36], which we consider to have low computational cost and briefly describe here.

Given a point $X$ on $\mathcal{M}_{n, p}$, the canonical metric $g^{c}$ on the tangent space $\mathcal{T}_{X} \mathcal{M}_{n, p}=$ $\left\{Z \in \mathbb{R}^{n, p}, Z^{\top} X+X^{\top} Z=0\right\}$ is defined as

$$
g^{c}\left(Z_{1}, Z_{2}\right):=\operatorname{tr}\left(Z_{1}^{\top}\left(I-\frac{1}{2} X X^{\top}\right) Z_{2}\right) .
$$

This metric considers the Stiefel manifold to be a quotient space $M_{n, p}=O_{n} / O_{n-p}$, in which $O_{k}$ is the group of $k \times k$ orthogonal matrices. Let us write $G_{i j}=\partial_{i j} \mathcal{F}$, then the gradient of $\mathcal{F}$ with respect to $g^{c}[1,36]$ is given by

$$
\nabla_{\mathcal{M}}^{c} \mathcal{F}(X)=G-X G^{\top} X
$$

Throughout the paper, we will always adopt the canonical metric, the superscript indicating the canonical metric will be omitted. Let $A=G X^{\top}-X G^{\top}$, the authors in [36] consider an implicit update scheme as

$$
Y(\tau)=X-\tau A\left(\frac{X+Y(\tau)}{2}\right) .
$$

This leads to the following Cayley transformation,

$$
Y(\tau)=\left(I+\frac{\tau}{2} A\right)^{-1}\left(I-\frac{\tau}{2} A\right) X .
$$

It has been shown in [36] that the update scheme automatically preserve the orthogonality constraint due to the property of Cayley transformation. In addition, with certain conditions, it has also been proved in [36] that the sequence generated using this algorithm satisfying $\lim _{k \rightarrow 0}\left\|\nabla F\left(X^{k}\right)\right\|_{F}=0$.

1.2. Global optimization by diffusions. For a general non-convex unconstrained optimization problem

$$
\min _{x \in \mathbb{R}^{n}} f(x) .
$$

A well known method is to consider the gradient flow

$$
\mathrm{d} x(t)=-\nabla f(x(t)) \mathrm{d} t,
$$

yet $x(t)$ is often trapped at a local stationary point due to nonconvexity. A well-known remedy is to add white noise to the gradient flow [3,6,9, 10], allowing the trajectory to "climb over the mountains" and escape from the local minimizers. Mathematically, this type of methods can be formulated as the following Stochastic Differential Equation (SDE) [24]:

$$
\mathrm{d} x(t)=-\nabla f(x(t)) \mathrm{d} t+\sigma(t) \mathrm{d} B(t),
$$

where $f(x)$ is the objective function defined on $\mathbb{R}^{n}$ and $B(t)$ is an $n$-dimensional standard Brownian motion, which is also known as the Wiener process. Different choices of $\sigma(t)$ lead to different diffusion algorithms and different results. It has been proved in $[6,9]$ that if the diffusion strength $\sigma(t)$ is chosen as $\sigma(t)=c / \sqrt{\log (t+2)}$ for some $c \geq c_{0}$, reffered as Continuous Diminishing Diffusion (CDD, also known as 
Simulated Annealing), $x(t)$ converges to the set of global minimizers under appropriate conditions on $f$. In [9], the objective function is defined on a compact set, while the assumption is lifted in [6]. Other choices of the diffusion strength $\sigma(t)$ are discussed in several articles. The properties with large $\sigma(t)$ is discussed in [37]. More recently, a method called intermittent diffusion (ID) has been proposed in [7], where a piecewise constant diffusion strength $\sigma(t)=\sum_{i=1}^{N} \sigma_{i} I_{\left[S_{i}, S_{i}+T_{i}\right]}(t)$ is considered. In other words, this method essentially considers to alternatively update variables between gradient descent and noisy gradient descent. It has been shown in [7] the global convergence ID and its effectiveness in specific problems.

To the best of our knowledge, only a few articles apply the SDE method to constrained problems. Problems with linear constrained is discussed in [29]. Portfolio selection having higher order moments with selected constraints is studied in [20]. The authors in [30] apply the method to robust chance constraint problems and a class of minimax problems is solved in [27]. In [28], problems with equality constraints are solved but no theoretical validation is provided that the constraints can be preserved using the proposed SDE.

1.3. Main Results. In order to find the global minimizers of orthogonality constrained problems, it is natural to consider a generalization of the diffusion methods based on (8) in Euclidean space to problems with orthogonality constraints (1). Our strategy is a combination of the CDD and ID, which leads to an optimization procedure that alternatively apply the diminishing diffusion and the deterministic local solver mentioned in subsection 1.1. We refer this procedure as an intermittent diminishing diffusion on manifold (IDDM). One crucial step of IDDM is to explore an computational tractable method to the SDE on the Stiefel manifold, which can be symbolically written as follows:

$$
\mathrm{d} X(t)=-\nabla_{\mathcal{M}} \mathcal{F}(X(t)) \mathrm{d} t+\sigma(t) \circ \mathrm{d} B_{\mathcal{M}}(t),
$$

where $\nabla_{\mathcal{M}}$ and $B_{\mathcal{M}}$ stand for gradient and Wiener process on manifold, respectively. One of the major challenges of using the above equation on the Stiefel manifold is the lack of global parameterization of the manifold, which make the numerical computation not straightforward to generate Wiener process on the Stiefel manifold. On the other hand, $\mathcal{M}_{n, p}$ is an embedding manifold in $\mathbb{R}^{n, p}$, whose embedding coordinates can be used to design an extrinsic form of the above SDE. In order to make use of (9) on numerical optimization, we propose an extrinsic presentation to facilitate numerical work. Our idea is to project the Brownian motion in the ambient space to the tangent space of $\mathcal{M}_{n, p}$. Based on this idea, we have theoretically validate the proposed procedure of IDDM for orthogonality constrained problems. More specifically, we have established the following results:

1. We theoretically show that the proposed extrinsic form is in fact generating feasible path constrained on $\mathcal{M}_{n, p}$.

2. We also validate that the proposed method of projection Brownian motion in $\mathbb{R}^{n, p}$ to the tangent space of $\mathcal{M}_{n, p}$ is an extrinsic form of the Brownian motion on the $\mathcal{M}_{n, p}$.

3. We further propose a numerical-efficient scheme to solve the proposed extrinsic equation and theoretically validate the half-order convergence of the scheme.

4. We also provide theoretical global convergence analysis of the proposed method, which is a consequence that the proposed extrinsic form satisfies the associated Fokker-Planck equation on the manifold. 
5. We numerically demonstrate that often only a few cycles of IDDM is needed to identify a better solution than the local algorithm for difficult problems with multiple local minimizers.

The rest of this paper is organized as follows. In section 2, we propose an extrinsic form of the SDE (9) and discuss its well-posedness. We also show the proposed extrinsic diffusion term in fact provides the Brownnian motion on the Steifel manifold. Numerical scheme of solving the proposed SDE and its convergence is discussed in section 3. After that, we describe the proposed intermittent diminishing diffusion on manifold (IDDM) and show that IDDM converges to global optimizers of the orthogonality constrained problems with probability almost equal to 1 in section 4 . Numerically we demonstrate the effectiveness of the proposed method on several applications involving orthogonality constrained optimization in section 5. Finally, we conclude our work in section 6 .

2. SDE on Stiefel manifold. In this section, we propose an explicit representation of the SDE (9). We also validate that the proposed explicit form is well-posed by showing that solutions of the explicit form stay on the Stiefel manifold with probability 1. We further show that the proposed method of projecting Brownian motion is a Brownian motion on the Stiefel manifold.

As we mentioned in the introduction, one crucial step of adapting SDE methods to the orthogonality constrained problems is how to design a computation tractable way of generating Brownian motion on the Stiefel manifold. Note that for any matrix $Z \in \mathbb{R}^{n, p}$, we can use the following operator to project $Z$ to $\mathcal{T}_{X} \mathcal{M}_{n, p}$.

$$
P: \mathbb{R}^{n \times p} \rightarrow \mathcal{T}_{X} \mathcal{M}_{n, p}, \quad Y \mapsto P_{X}(Z)=Z-\alpha X Z^{\top} X-\beta X X^{\top} Z
$$

where $\alpha=\sqrt{2} / 2, \beta=1-\sqrt{2} / 2$. This motivates us to project the Brownian motion in the ambient space to the tangent space of $\mathcal{M}_{n, p}$ based on this projection operator. Namely, we propose the extrinsic representation of the SDE (9) on Stiefel manifold as

$$
\mathrm{d} X(t)=-\nabla_{\mathcal{M}} \mathcal{F}(X(t)) \mathrm{d} t+\sigma(t) \sum_{u=1}^{n} \sum_{v=1}^{p} P_{u v}(X(t)) \circ \mathrm{d} B_{u v}(t),
$$

where $\left\{B_{u v}(t)\right\}$ is a series of (independent) one-dimensional standard Brownian motion, and $P_{u v}$ is defined by

$$
\begin{array}{r}
P_{u v}(X)=E_{u v}-\alpha X E_{u v}^{\top} X-\beta X X^{\top} E_{u v}, \quad X \in \mathcal{M}_{n, p}, \\
u=1,2, \ldots, n, \quad v=1,2, \ldots, p .
\end{array}
$$

2.1. Well-posedness of the extrinsic SDE. There are several issues with respect to (11) to be clarified. First, the definition of coefficients of drift term and diffusion term is restricted to the manifold, and thus a proper extension is needed in order to make it a well-posed SDE in Euclidean space $\mathbb{R}^{n \times p}$. We first show that the SDE given by (11) is well-posed. In other words, there is an equivalent extension to the euclidean space $\mathbb{R}^{n \times p}$ that exists, lies on the manifold and gives a unique solution. We also expect that the solution will not leave the manifold so that the off-manifold coefficient will not impact the solution. The answers of all the above concerns are addressed in the following theorem.

THEOREM 1. Let $V$ be an arbitrary smooth vector field on the Stiefel manifold $\mathcal{M}_{n, p}$. Then 
(a) There exists some smooth extensions of $V(X)$ and $P_{u v}(X)$ in $\mathbb{R}^{n \times p}$ (denoted by $\tilde{V}(X)$ and $\left.\tilde{P}_{u v}(X)\right)$, which are globally Lipschitz. Hence, there exists a unique solution $X(t, w)$ for the extended $S D E$

$$
\mathrm{d} X(t)=\tilde{V}(X(t)) \mathrm{d} t+\sigma(t) \sum_{u=1}^{n} \sum_{v=1}^{p} \tilde{P}_{u v}(X(t)) \circ \mathrm{d} B_{u v}(t)
$$

in $\mathbb{R}^{n \times p}$ once the extension is fixed.

(b) Let $X(t)$ be the solution of (13), and then $X(t)$ almost surely stays on $\mathcal{M}_{n, p}$ provided it originate on the manifold, i.e.,

$$
\mathbb{P}\left\{X(t) \in \mathcal{M}_{n, p} \mid X(0) \in \mathcal{M}_{n, p}\right\}=1, \quad \forall t \geq 0 .
$$

In addition, $X(t)$ does not leave its connected component in the case of $n=p$, i.e.,

$$
\mathbb{P}\left\{\operatorname{det}(X(t))=\operatorname{det}(X(0)) \mid X(0) \in \mathcal{M}_{n, n}\right\}=1, \quad \forall t \geq 0 .
$$

The solution of (13) is unique regardless of the extension of $V$ and $P_{u v}$.

Proof. (a) Direct observation suggests that $\mathcal{M}_{n, p}$ is a compact subset of $\mathbb{R}^{n \times p}$, which makes it possible to construct a globally Lipschitz extension. The extension is not unique, and for example we can take

$$
\left\{\begin{array}{l}
\tilde{V}(X):=\zeta_{\varepsilon}\left(\left\|X^{\top} X-I_{p}\right\|_{2}^{2}\right) V(\mathcal{Q}(X)) \\
\tilde{P}_{u v}(X):=\zeta_{\varepsilon}\left(\left\|X^{\top} X-I_{p}\right\|_{2}^{2}\right)\left(E_{u v}-\alpha X E_{u v}^{\top} X-\beta X X^{\top} E_{u v}\right),
\end{array}\right.
$$

where $\mathcal{Q}(X)$ indicates the $n$-by- $p$ matrix from reduced $Q R$ decomposition of $X$ (here we follow the convention that the diagonal entries of upper triangular $R$ are non-negative). $\zeta_{\varepsilon}$ is a $C_{0}^{\infty}([0,+\infty))$ mollifier satisfying

$$
\zeta_{\varepsilon}([0, \varepsilon]) \equiv 1, \quad \zeta_{\varepsilon}([2 \varepsilon,+\infty)) \equiv 0,
$$

where $\varepsilon$ is a given positive constant with $\varepsilon<1 / 2$. Under this condition one can show that both $\tilde{V}$ and $\tilde{P}_{i j}$ are globally Lipschitz. The existence and uniqueness of (13) follow directly from the existence and uniqueness theorem of general SDE (see Theorem 5.2.1 of [24] for example).

(b) The general feasibility results (14) can be derived by viewing (13) as a process driven by $\mathbb{R}^{n p+1}$-valued semimartingale $Z(t)=\left(t, \sigma(t) B_{i j}(t)\right)$ and applying Proposition 1.2.8 of [14]. The special case of $n=p$ can be treated similarly but viewing two connected components as two separate manifolds instead. The uniqueness can be referred to Theorem 1.2.9 of [14].

In view of the uniqueness result we can specify that the extension of $\tilde{V}$ and $\tilde{P}_{u v}$ is given by (15) to facilitate further discussion. Sometimes it would be more convenient to analyze the Ito version of (13), which can be derived from the following transformation property between Ito SDE and Stratonovich SDE in the Euclidean space.

Lemma 2 ([24]). The coresponding Ito version of Stratonovich system

$$
\mathrm{d} X_{\eta}(t)=h_{\eta}(X(t), t) \mathrm{d} t+\sum_{\lambda} H_{\eta \lambda}(X(t), t) \circ \mathrm{d} B_{\lambda}(t)
$$


is given by

$$
\mathrm{d} X_{\eta}(t)=\left[h_{\eta}(X(t), t)+\frac{1}{2} \sum_{\lambda}\left(\sum_{\mu} \frac{\partial H_{\eta \lambda}}{\partial X_{\mu}} H_{\mu \lambda}\right)\right] \mathrm{d} t+\sum_{\lambda} H_{\eta \lambda}(X(t), t) \mathrm{d} B_{\lambda}(t)
$$

Based on this lemma, we can derive the Ito version of the Stratonovich SDE (13) described in the following theorem.

THEOREM 3. The corresponding Ito version of Stratonovich SDE (13) on $\mathcal{M}_{n, p}$ (with feasible initial point $X(0) \in \mathcal{M}_{n, p}$ ) is given by

$$
\begin{aligned}
\mathrm{d} X(t) & =\left(V(X(t))-\frac{n-1}{2} \sigma^{2}(t) X(t)\right) \mathrm{d} t \\
& +\sigma(t) \sum_{u=1}^{n} \sum_{v=1}^{p}\left(E_{u v}-\alpha X E_{u v}^{\top} X-\beta X X^{\top} E_{u v}\right) \mathrm{d} B_{u v}(t)
\end{aligned}
$$

Here we omit the discussion of definition of parameters outside the manifold.

Proof. Write (13) coordinate-wise as

$$
\begin{aligned}
\mathrm{d} X_{i j}(t)= & \tilde{V}_{i j}(X) \mathrm{d} t+\sigma(t) \sum_{u=1}^{n} \sum_{v=1}^{p}\left[\zeta_{\varepsilon}\left(\left\|X^{\top} X-I_{p}\right\|_{2}^{2}\right)\right. \\
& \left.\left(\delta_{i u} \delta_{j v}-\alpha X_{i v} X_{u j}-\beta \sum_{w=1}^{p} X_{i w} X_{u w} \delta_{j v}\right) \circ \mathrm{d} B_{u v}(t)\right] .
\end{aligned}
$$

Applying Lemma 2 by viewing the index $\eta=(i, j)$ and $\lambda=(u, v)$, one can show that the $(i, j)$-th entry of the additional drift term is given by

$$
\begin{aligned}
& \frac{1}{2} \sigma^{2}(t) \sum_{u, v, s, t}\left[\partial_{s t}\left(-\alpha X_{i v} X_{u j}-\beta \sum_{w=1}^{p} X_{i w} X_{u w} \delta_{j v}\right)\right. \\
& \left.\cdot\left(\delta_{u s} \delta_{v t}-\alpha X_{s v} X_{u t}-\beta \sum_{w}^{p} X_{s w} X_{u w} \delta_{v t}\right)\right] \\
= & \frac{1}{2} \sigma^{2}(t) \sum_{u, v, s, t}\left[\left(-\alpha \delta_{i s} \delta_{v t} X_{u j}-\alpha \delta_{u s} \delta_{j t} X_{i v}-\beta \delta_{i s} \delta_{j v} X_{u t}-\beta \delta_{j v} \delta_{u s} X_{i t}\right)\right. \\
& \left.\cdot\left(\delta_{u s} \delta_{v t}-\alpha X_{s v} X_{u t}-\beta \sum_{w=1}^{p} X_{s w} X_{u w} \delta_{v t}\right)\right] \\
= & \frac{1}{2} \sigma^{2}(t)\left[\left(2 \alpha^{2}+\beta^{2}+\alpha \beta-\beta\right)-(\alpha+\beta) n+\left(\beta^{2}+3 \alpha \beta-\alpha\right) p\right] X_{i j} \\
= & -\frac{(n-1)}{2} \sigma^{2}(t) X_{i j} .
\end{aligned}
$$

Here we omit the discussion of coefficients off the manifold as the derivation of the mollifier $\eta_{\varepsilon}$ will not affect the on-manifold result due to the hypothesis of (16). In addition, the second last equality of the above derivation is provided by simply expanding each item of (19) using the facts $\sum_{u} X_{u i} X_{u j}=\delta_{i j}$ and $\sum_{u v} X_{u v}^{2}=p$. More specifically, we summarize products among pairs in the following table: 


\begin{tabular}{|c|c|c|c|}
\hline$\times$ & $\delta_{u s} \delta_{v t}$ & $-\alpha X_{s v} X_{u t}$ & $-\beta \sum_{w=1}^{p} X_{s w} X_{u w} \delta_{v t}$ \\
\hline$-\alpha \sum_{u, v, s, t} \delta_{i s} \delta_{v t} X_{u j}$ & $-\alpha p X_{i j}$ & $\alpha^{2} X_{i j}$ & $\alpha \beta p X_{i j}$ \\
\hline$-\alpha \sum_{u, v, s, t} \delta_{u s} \delta_{j t} X_{i v}$ & $-\alpha n X_{i j}$ & $\alpha^{2} X_{i j}$ & $\alpha \beta p X_{i j}$ \\
\hline$-\beta \sum_{u, v, s, t} \delta_{i s} \delta_{j v} X_{u t}$ & $-\beta X_{i j}$ & $\alpha \beta p X_{i j}$ & $\beta^{2} X_{i j}$ \\
\hline$-\beta \sum_{u, v, s, t} \delta_{j v} \delta_{u s} X_{i t}$ & $-\beta n X_{i j}$ & $\alpha \beta X_{i j}$ & $\beta^{2} p X_{i j}$ \\
\hline
\end{tabular}

REMARK 4. Using the projection operator defined in (10), we can simplify the notation by writing the diffusion term in short as

$$
\sum_{u=1}^{n} \sum_{v=1}^{p}\left(E_{u v}-\alpha X E_{u v}^{\top} X-\beta X X^{\top} E_{u v}\right) \mathrm{d} B_{u v}(t)=P_{X}(\mathrm{~d} B(t)) .
$$

For example, the above Ito SDE (18) can be simplified as

$$
\mathrm{d} X(t)=\left(V(X(t))-\frac{n-1}{2} \sigma^{2}(t) X(t)\right) \mathrm{d} t+\sigma(t) P_{X}(\mathrm{~d} B(t)) .
$$

We will follow this convention throughout the paper.

2.2. Laplace-Beltrami Operator on Canonical Stiefel Manifold. It remains to show that the diffusion term of extrinsic SDE (13) is the Brownian motion on the Stiefel manifold. Before considering that, we first provide an extrinsic representation of the Laplace-Beltrami (LB) operator on the Stiefel manifold.

Theorem 5 (Extrinsic form of the LB operator on $\mathcal{M}_{n, p}$ ). The LB operator at $X$ on $\mathcal{M}_{n, p}$ (endowed with the canonical metric) is given by

$$
\Delta_{\mathcal{M}_{n, p}}=\sum_{i=1}^{n} \sum_{j=1}^{p} \partial_{i j}^{2}-\sum_{i, u=1}^{n} \sum_{j, v=1}^{p} X_{i v} X_{u j} \partial_{i j} \partial_{u v}-(n-1) \sum_{i=1}^{n} \sum_{j=1}^{p} X_{i j} \partial_{i j} .
$$

We calculate the LB operator using the trace of the Hessian operator along an orthonormal basis in the tangent space $\mathcal{T}_{X} \mathcal{M}_{n, p}$. First, we provide an orthonormal basis in the following lemma.

Lemma 6 (Orthonormal basis of $\mathcal{T}_{X} \mathcal{M}_{n, p}$ ). Let $Q$ to be an extended orthogonal matrix of $X$, such that $Q \in \mathbb{R}^{n \times n}, Q^{\top} Q=I_{n}$ and $Q=\left[X, X_{\perp}\right]$. An orthonormal basis of $\mathcal{T}_{X} \mathcal{M}_{n, p}$ is given by

$$
\begin{aligned}
U_{i j} & =Q\left(E_{i j}-E_{j i}\right), & i<j \leq p, \\
U_{i j} & =Q E_{i j}, & i>p,
\end{aligned}
$$

where $E_{i j}$ is the element matrix in $\mathbb{R}^{n \times p}$. The set of the basis is denoted by $\Lambda$.

Proof of Lemma 6. To simplify the notation, we set $\tilde{E}_{i j}=E_{i j}-E_{j i},(i, j \leq p)$ or $\tilde{E}_{i j}=E_{i j},(i>p)$. The orthogonality of $Q$ indicates that $\Lambda$ is linear independent. It can be easily calculated that $\Lambda$ has $n p-p(p+1) / 2$ elements so that $\Lambda$ spans $\mathcal{T}_{X} \mathcal{M}_{n, p}$. We next calculate the inner product as

$$
\begin{aligned}
& g^{c}\left(U_{i j}, U_{k l}\right)=\operatorname{tr}\left(\tilde{E}_{i j}^{\top} \operatorname{diag}\left\{\frac{1}{2} I_{p}, I_{n}\right\} \tilde{E}_{k l}\right)=0, \quad \text { if }(i, j) \neq(k, l) \text { and }(j, i) \neq(k, l) ; \\
& g^{c}\left(U_{i j}, U_{i j}\right)=\operatorname{tr}\left(\tilde{E}_{i j}^{\top} \operatorname{diag}\left\{\frac{1}{2} I_{p}, I_{n}\right\} \tilde{E}_{i j}\right)=1, \quad \text { if } i \neq j ;
\end{aligned}
$$


The results above indicate that $\Lambda$ is the set of orthonormal basis of $\mathcal{T}_{X} \mathcal{M}_{n, p}$. From the orthonormal basis we can calculate the LB operator $\Delta_{\mathcal{M}_{n, p}}$.

Proof of Theorem 5. It has been shown in [8] that

$$
\begin{aligned}
\nabla_{\mathcal{M}_{n, p}}^{2} \mathcal{F}\left(Z_{1}, Z_{2}\right) & =\nabla_{E}^{2} \mathcal{F}\left(Z_{1}, Z_{2}\right)+\frac{1}{2} \operatorname{tr}\left(G^{\top} Z_{1} X^{\top} Z_{2}+X^{\top} Z_{1} G^{\top} Z_{2}\right) \\
& -\frac{1}{2} \operatorname{tr}\left(\left(X^{\top} G+G^{\top} X\right) Z_{1}^{\top}\left(I-X X^{\top}\right) Z_{2}\right), Z_{1}, Z_{2} \in \mathcal{T}_{X} \mathcal{M}_{n, p}
\end{aligned}
$$

where $\nabla_{E}^{2}$ is the Hessian operator in the Euclidean space $\mathbb{R}^{n \times p}$ and $G_{i j}=\partial_{i j} \mathcal{F}$.

From the orthonormal basis we obtain

$$
\begin{aligned}
\nabla_{\mathcal{M}_{n, p}}^{2} \mathcal{F}\left(U_{i j}, U_{i j}\right) & =\nabla_{E}^{2} \mathcal{F}\left(Q \tilde{E}_{i j}, Q \tilde{E}_{i j}\right)+\operatorname{tr}\left(G^{\top} Q \tilde{E}_{i j} X^{\top} Q \tilde{E}_{i j}\right) \\
& -\frac{1}{2} \operatorname{tr}\left(\left(X^{\top} G+G^{\top} X\right) \tilde{E}_{i j}^{\top} Q^{\top}\left(I-X X^{\top}\right) Q \tilde{E}_{i j}\right) \\
& =\nabla_{E}^{2} \mathcal{F}\left(Q \tilde{E}_{i j}, Q \tilde{E}_{i j}\right)+\operatorname{tr}\left(G^{\top} Q \tilde{E}_{i j}\left[I_{p}, 0_{p \times(n-p)}\right] \tilde{E}_{i j}\right) \\
& -\frac{1}{2} \operatorname{tr}\left(\left(X^{\top} G+G^{\top} X\right) \tilde{E}_{i j}^{\top} \operatorname{diag}\left\{0_{p}, I_{n-p}\right\} \tilde{E}_{i j}\right) .
\end{aligned}
$$

Hence, we have

$$
\begin{aligned}
\Delta_{\mathcal{M}_{n, p}} \mathcal{F} & =\sum_{U_{i j} \in \Lambda} \nabla_{\mathcal{M}_{n, p}}^{2} \mathcal{F}\left(U_{i j}, U_{i j}\right) \\
& =\sum_{i>p}\left[\nabla_{E}^{2} \mathcal{F}\left(Q E_{i j}, Q E_{i j}\right)+0-\frac{1}{2} \operatorname{tr}\left(\left(X^{\top} G+G^{\top} X\right) E_{j j}^{p}\right)\right] \\
& +\sum_{i<j \leq p}\left[\nabla_{E}^{2} \mathcal{F}\left(Q \tilde{E}_{i j}, Q \tilde{E}_{i j}\right)+\operatorname{tr}\left(G^{\top} Q \tilde{E}_{i j}\left[I_{p}, 0_{p \times(n-p)}\right] \tilde{E}_{i j}\right)-0\right] \\
= & \sum_{i>p}\left[\nabla_{E}^{2} \mathcal{F}\left(Q E_{i j}, Q E_{i j}\right)-\left(G^{\top} X\right)_{j j}\right] \\
& +\sum_{i<j \leq p}\left[\left(\nabla_{E}^{2} \mathcal{F}\left(Q E_{i j}, Q E_{i j}\right)+\nabla_{E}^{2} \mathcal{F}\left(Q E_{j i}, Q E_{j i}\right)-2 \nabla_{E}^{2} \mathcal{F}\left(Q E_{i j}, Q E_{j i}\right)\right)\right. \\
& \left.-\left(\left(G^{\top} X\right)_{i i}+\left(G^{\top} X\right)_{j j}\right)\right] \\
& =\sum_{i, j} \nabla_{E}^{2} F\left(Q E_{i j}, Q E_{i j}\right)-\sum_{i, j \leq p} \nabla_{E}^{2} F\left(Q E_{i j}, Q E_{j i}\right)-(n-1) \operatorname{tr}\left(G^{\top} X\right) .
\end{aligned}
$$

Orthogonal $Q$ indicates that the linear transformation $V \in \mathbb{R}^{n \times p} \mapsto Q V \in \mathbb{R}^{n \times p}$ is orthogonal (under Euclidean metric). Therefore,

$$
\begin{aligned}
\Delta_{\mathcal{M}_{n, p}} \mathcal{F} & =\sum_{i, j} \nabla_{E}^{2} F\left(Q E_{i j}, Q E_{i j}\right)-\sum_{i, j \leq p} \nabla_{E}^{2} F\left(Q E_{i j}, Q E_{j i}\right)-(n-1) \operatorname{tr}\left(G^{\top} X\right) \\
& =\Delta_{E} \mathcal{F}-\sum_{i, j \leq p} \nabla_{E}^{2} \mathcal{F}\left(X E_{i j}^{p}, X E_{j i}^{p}\right)-(n-1) \operatorname{tr}\left(G^{\top} X\right),
\end{aligned}
$$

where $E_{i j}^{p}$ is the element matrix in $\mathbb{R}^{p \times p}$. With some expansion and mark changing, we obtain

$$
\Delta_{\mathcal{M}_{n, p}}=\sum_{i=1}^{n} \sum_{j=1}^{p} \partial_{i j}^{2}-\sum_{i, u=1}^{n} \sum_{j, v=1}^{p} X_{i v} X_{u j} \partial_{i j} \partial_{u v}-(n-1) \sum_{i=1}^{n} \sum_{j=1}^{p} X_{i j} \partial_{i j},
$$

which completes the proof. 


\subsection{Extrinsic formulation of Brownian motion on the Stiefel Manifold.}

We now show that the diffusion term introduced in (13) is exactly the $\mathcal{M}$-valued Brownnian motion driven by half of the Laplace-Beltrami operator. We state the result in the following theorem.

Theorem 7. Suppose that $W(t)$ is the solution of the following SDE

$$
\mathrm{d} W(t)=\sum_{u=1}^{n} \sum_{v=1}^{p}\left(E_{u v}-\alpha W E_{u v}^{\top} W-\beta W W^{\top} E_{u v}\right) \circ \mathrm{d} B_{u v}(t) .
$$

Then $W(t)$ is driven by half of Laplacian-Beltrami operator $\Delta_{\mathcal{M}_{n, p}}$ on Stiefel Manifold, i.e.,

$$
\frac{1}{2} \Delta_{\mathcal{M}_{n, p}} \varphi(W(t))=\mathcal{L} \varphi(W(t)):=\lim _{t \rightarrow 0+} \frac{\mathbb{E}\left[\varphi(W(t)) \mid W(0)=w_{0}\right]-\varphi\left(w_{0}\right)}{t} .
$$

Proof. From Theorem 3, the Ito version of (24) is

$$
\mathrm{d} W(t)=-\frac{n-1}{2} X \mathrm{~d} t+\sum_{u=1}^{n} \sum_{v=1}^{p}\left(E_{u v}-\alpha W E_{u v}^{\top} W-\beta W W^{\top} E_{u v}\right) \mathrm{d} B_{u v}(t) .
$$

The generator of $\varphi$ can be derived as [24]

$$
\begin{aligned}
& \mathcal{L} \varphi=-\frac{n-1}{2} \sum_{i=1}^{n} \sum_{j=1}^{p} X_{i j} \partial_{i j} \varphi+\frac{1}{2} \sum_{i, u, s}^{n} \sum_{j, v, t}^{p}\left(\delta_{i s} \delta_{j t}-\alpha X_{i t} X_{s j}-\beta \sum_{w}^{p} X_{i w} X_{s w} \delta_{t j}\right) \\
& \cdot\left(\delta_{u s} \delta_{v t}-\alpha X_{u t} X_{s v}-\beta \sum_{z}^{p} X_{u z} X_{s z} \delta_{t v}\right) \partial_{i j} \partial_{u v} \varphi
\end{aligned}
$$

We expand pairwise products in the second term of the above equation as follows:

$$
\begin{gathered}
\sum_{i, j, u, v, s, t}\left(\delta_{i s} \delta_{j t}\right)\left(\delta_{u s} \delta_{v t}\right)\left(\partial_{i j} \partial_{u v} \varphi\right)=\sum_{i, j} \partial_{i j}^{2} \varphi \\
\sum_{i, j, u, v, s, t}\left(\delta_{i s} \delta_{j t}\right)\left(-\alpha X_{u t} X_{s v}\right)\left(\partial_{i j} \partial_{u v} \varphi\right)=-\alpha \sum_{i, j, u, v} X_{u j} X_{i v}\left(\partial_{i j} \partial_{u v} \varphi\right),(\text { twice }) \\
\sum_{i, j, u, v, s, t}\left(\delta_{i s} \delta_{j t}\right)\left(-\beta \sum_{z=1}^{p} X_{u z} X_{s z} \delta_{t v}\right)\left(\partial_{i j} \partial_{u v} \varphi\right)=-\beta \sum_{i, j, u, v} X_{i v} X_{u v}\left(\partial_{i j} \partial_{u j} \varphi\right), \text { (twice) } \\
\sum_{i, j, u, v, s, t}\left(-\alpha X_{i t} X_{s j}\right)\left(-\alpha X_{u t} X_{s v}\right)\left(\partial_{i j} \partial_{u v} \varphi\right)=\alpha^{2} \sum_{i, j, u, v} X_{i v} X_{u v}\left(\partial_{i j} \partial_{u j} \varphi\right) \\
\sum_{i, j, u, v, s, t}\left(-\alpha X_{i t} X_{s j}\right)\left(-\beta \sum_{z=1}^{p} X_{u z} X_{s z} \delta_{t v}\right)\left(\partial_{i j} \partial_{u v} \varphi\right)=\alpha \beta \sum_{i, j, u, v} X_{i v} X_{u j}\left(\partial_{i j} \partial_{u v} \varphi\right),(\text { twice }) \\
\sum_{i, j, u, v, s, t}\left(-\beta \sum_{w=1}^{p} X_{i w} X_{s w} \delta_{t j}\right)\left(-\beta \sum_{z=1}^{p} X_{u z} X_{s z} \delta_{t v}\right)\left(\partial_{i j} \partial_{u v} \varphi\right) \\
=\beta^{2} \sum_{i, j, u}\left(X X^{\top} X X^{\top}\right)_{i u}\left(\partial_{i j} \partial_{u j} \varphi\right)=\beta^{2} \sum_{i, j, u, v} X_{i v} X_{u v}\left(\partial_{i j} \partial_{u j} \varphi\right)
\end{gathered}
$$


Hence, we have

$$
\begin{aligned}
\mathcal{L} \varphi= & -\frac{n-1}{2} \sum_{i=1}^{n} \sum_{j=1}^{p} X_{i j} \partial_{i j} \varphi+\frac{1}{2}\left[\sum_{i, j} \partial_{i j}^{2} \varphi+(-2 \alpha+2 \alpha \beta) \sum_{i, j, u, v} X_{i v} X_{u j} \partial_{i j} \partial_{u v} \varphi\right. \\
& \left.+\left(-2 \beta+\alpha^{2}+\beta^{2}\right)\left(\sum_{i, j, u, v} X_{i v} X_{u v} \partial_{i j} \partial_{u j} \varphi\right)\right] .
\end{aligned}
$$

Substituting $\alpha=\sqrt{2} / 2$ and $\beta=1-\sqrt{2} / 2$ we obtain

$$
\mathcal{L} \varphi=-\frac{n-1}{2} \sum_{i=1}^{n} \sum_{j=1}^{p} X_{i j} \partial_{i j} \varphi+\frac{1}{2}\left[\sum_{i, j} \partial_{i j}^{2} \varphi-\sum_{i, j, u, v} X_{i v} X_{u j} \partial_{i j} \partial_{u v} \varphi\right]=\frac{1}{2} \Delta_{\mathcal{M}_{n, p}} \varphi .
$$

The next corollary is a direct extension of the above theorem.

COROLlary 8. The Fokker-Planck Equation of (11) is given by

$$
\frac{\partial p}{\partial t}=-\nabla_{\mathcal{M}_{n, p}} \cdot\left(p \nabla_{\mathcal{M}_{n, p}} \mathcal{F}\right)+\frac{1}{2} \sigma^{2}(t) \Delta_{\mathcal{M}_{n, p}} p,
$$

where $\nabla_{\mathcal{M}_{n, p}}, \nabla_{\mathcal{M}_{n, p}}, \Delta_{\mathcal{M}_{n, p}}$ represent the gradient, divergence and Laplace-Beltrami operator on the Stiefel manifold endowed with canonical metric, respectively.

3. Numerical scheme of the SDE and its convergence. We next provide a numerical scheme to solve the SDE (11). Our idea is first projecting the random noise in the ambient space to the tangent space of the Stiefel manifold. After that, we apply the Cayley transformation similar as the method discussed in [36]. More precisely, we propose the following update scheme to solve the SDE (11):

$$
\left\{\begin{array}{l}
Z_{k}=-\delta_{k} G_{k}+\sigma_{k}\left(I_{n}-\beta Y_{k} Y_{k}^{\top}\right) \delta B_{k}, \\
A_{k}=Z_{k} Y_{k}^{\top}-Y_{k} Z_{k}^{\top} \\
Y_{k+1}=\left(I-\frac{A_{k}}{2}\right)^{-1}\left(I+\frac{A_{k}}{2}\right) Y_{k} .
\end{array}\right.
$$

In the case of $p=n$, we have a simpler form

$$
Z_{k}=-\delta_{k} G_{k}+\alpha \sigma_{k} \delta B_{k}
$$

In the spherical constrained case of $p=1$, we can show that

$$
A_{k}=\left(-\delta_{k} G_{k}+\sigma_{k} \delta B_{k}\right) Y_{k}^{\top}-Y_{k}\left(-\delta_{k} G_{k}+\sigma_{k} \delta B_{k}\right)^{\top} .
$$

We point out that there is an efficient way to compute $Y_{k+1}$ in the case of $p<$ $n / 2$ or $p=1$ based on the Sherman-Morrison-Woodbury formula similar to the way discussed in [36].

Lemma 9 ([36]).

(1) Rewrite $A_{k}=U_{k} V_{k}^{\top}$ for $U_{k}=\left[Z_{k}, Y_{k}\right]$ and $V_{k}=\left[Y_{k},-Z_{k}\right]$. If $I-\frac{1}{2} V_{k}^{\top} U_{k}$ is invertible, then

$$
Y_{k+1}=Y_{k}+U_{k}\left(I-\frac{1}{2} V_{k}^{\top} U_{k}\right)^{-1} V_{k}^{\top} Y_{k}
$$


(2) For the vector case,

$$
Y_{k+1}=Y_{k}+\frac{Z_{k}}{1-\left(\frac{1}{2}\right)^{2}\left(Z_{k}^{\top} Y_{k}\right)^{2}+\left(\frac{1}{2}\right)^{2} Z_{k}^{\top} Z_{k}}-\frac{Z_{k}^{\top} Y_{k}-\frac{1}{2}\left(\left(Z_{k}^{\top} Y_{k}\right)^{2}\right)+Z_{k}^{\top} Z_{k}}{1-\left(\frac{1}{2}\right)^{2}\left(Z_{k}^{\top} Y_{k}\right)^{2}+\left(\frac{1}{2}\right)^{2} Z_{k}^{\top} Z_{k}} Y_{k} .
$$

The numerical scheme can now be summarized in Algorithm 1.

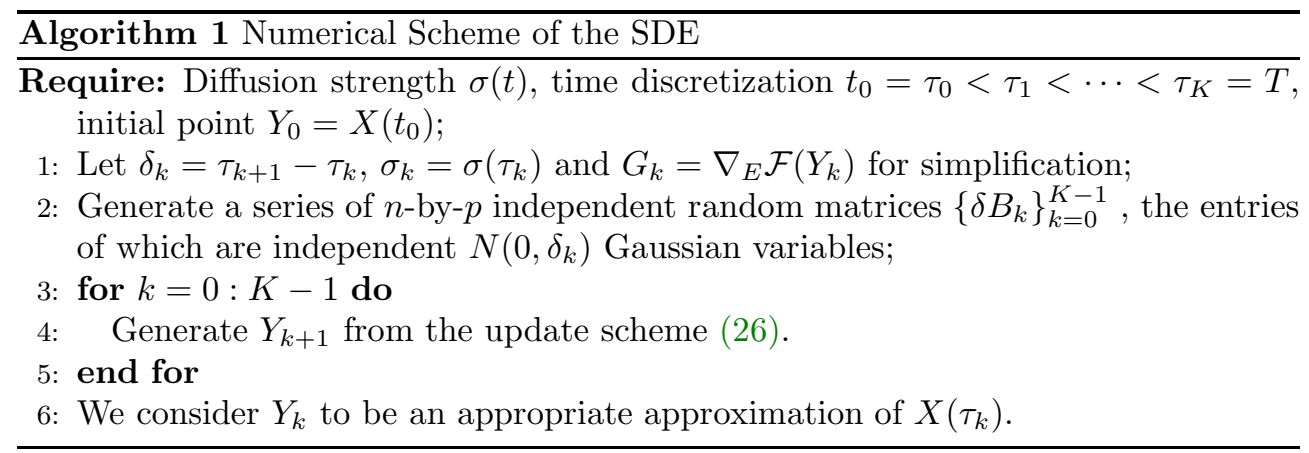

Now we state the strong convergence result. For simplicity, we state and prove the result in the case of constant $\sigma(t) \equiv \sigma_{0}$. Similar result can be proved with variational $\sigma(t)$ under trivial changes.

Theorem 10 (Half Order Strong Convergence). Denote $X(T)$ as a solution of the $S D E$ (11) and let $\delta=\max _{k}\left\{\delta_{k}\right\}$. Then there exists a positive constant $C=C(T)$ independent of $\delta$, as well as a constant $\delta_{0}>0$ such that

$$
\mathbb{E}\left\|X(T)-Y_{K}\right\|_{2}^{2} \leq C \delta, \quad \forall \delta \in\left(0, \delta_{0}\right)
$$

Proof. Without loss of generality, we suppose $t_{0}=0$. For $0=t_{0} \leq t \leq T$, we define

$$
R(t):=\sup _{0 \leq s \leq t} \mathbb{E}\left\|X(s)-Y_{k_{s}}\right\|_{F}^{2},
$$

where $k_{t}$ is the largest integer $k$ for which $\tau_{k}$ does not exceed $t$, i.e.,

$$
k_{t}:=\max \left\{k=0,1, \ldots, K: \tau_{k} \leq t\right\}
$$

Rewriting (11) into an Ito integral form yields

$$
X(s)-X(0)=\int_{0}^{s}\left[-\nabla_{\mathcal{M}} f(X(\tau))-\frac{n-1}{2} \sigma_{0}^{2} X(\tau)\right] \mathrm{d} \tau+\sigma_{0} \int_{0}^{s} \sum_{u, v} P_{u v}(X(\tau)) \mathrm{d} B(\tau)
$$


Substituting into (30) and applying the Schwarz inequality yields

$$
\begin{aligned}
& R(t)=\sup _{0 \leq s \leq t} \mathbb{E} \| \sum_{k=0}^{k_{s}-1}\left(Y_{k+1}-Y_{k}\right) \\
& +\int_{0}^{s}\left[\nabla_{\mathcal{M}} \mathcal{F}(X(\tau))+\frac{n-1}{2} \sigma_{0}^{2} X(\tau)\right] \mathrm{d} \tau-\sigma_{0} \int_{0}^{s} P_{X(\tau)}(\mathrm{d} B(\tau)) \|_{F}^{2} \\
& \leq 7 \sup _{0 \leq s \leq t}\left\{\mathbb{E}\left\|\sum_{k=0}^{k_{s}-1}\left[\mathbb{E}\left(Y_{k+1}-Y_{k} \mid Y_{k}\right)-\delta_{k}\left(-\nabla_{\mathcal{M}} \mathcal{F}\left(Y_{k}\right)-\frac{n-1}{2} \sigma_{0}^{2} Y_{k}\right)\right]\right\|_{F}^{2}\right. \\
& +\mathbb{E}\left\|\sum_{k=0}^{k_{s}-1}\left[Y_{k+1}-Y_{k}-\mathbb{E}\left(Y_{k+1}-Y_{k} \mid Y_{k}\right)-\sigma_{0} P_{Y_{k}}\left(\delta B_{k}\right)\right]\right\|_{F}^{2} \\
& +\mathbb{E}\left\|\sum_{k=0}^{k_{s}-1}\left[\int_{\tau_{k}}^{\tau_{k+1}}\left[\nabla_{\mathcal{M}} \mathcal{F}(X(\tau))\right] \mathrm{d} \tau-\delta_{k} \nabla_{\mathcal{M}} \mathcal{F}\left(Y_{\tau_{k}}\right)\right]\right\|_{F}^{2} \\
& +\mathbb{E}\left\|\sum_{k=0}^{k_{s}-1}\left[\int_{\tau_{k}}^{\tau_{k+1}}\left(\frac{n-1}{2} \sigma_{0}^{2} X(\tau)\right) \mathrm{d} \tau-\delta_{k}\left(\frac{n-1}{2} \sigma_{0}^{2} Y_{\tau_{k}}\right)\right]\right\|_{F}^{2} \\
& +\mathbb{E}\left\|\sigma_{0}^{k_{s}-1}\left[\int_{k=0}^{\tau_{k+1}} P_{\tau_{k}}(\mathrm{~d} B(\tau))-P_{Y_{k}}\left(\delta B_{k}\right)\right]\right\|_{F}^{2} \\
& +\mathbb{E}\left\|\int_{\tau_{k_{s}}}^{s}\left(\nabla_{\mathcal{M}} \mathcal{F}(X(\tau))+\frac{n-1}{2} \sigma_{0}^{2} X(\tau)\right) \mathrm{d} \tau\right\|_{F}^{2} \\
& \left.+\mathbb{E}\left\|\sigma_{0}^{s} \int_{\tau_{k_{s}}}^{s} P_{X(\tau)}(\mathrm{d} B(\tau))\right\| \|_{F}^{2}\right\}
\end{aligned}
$$

We next analyze the seven terms of (31) in order. Iterating (26) repeatedly yields

$$
Y_{k+1}-Y_{k}=A_{k} Y_{k}+\frac{1}{2} A_{k}^{2} Y_{k}+\frac{1}{8} A_{k}^{3}\left(Y_{k}+Y_{k+1}\right) .
$$

Direct calculus shows that

$$
\begin{aligned}
A_{k} Y_{k} & =Z_{k}-Y_{k} Z_{k}^{\top} Y_{k} \\
& =-\delta_{k}\left(G_{k}-Y_{k} G_{k}^{\top} Y_{k}\right)+\sigma_{0}\left[\left(I-\beta Y_{k} Y_{k}^{\top}\right) \delta B_{k}-Y_{k} \delta B_{k}^{\top}\left(I-\beta Y_{k} Y_{k}^{\top}\right) Y_{k}\right] \\
& =-\delta_{k}\left(\nabla_{\mathcal{M}} \mathcal{F}\left(Y_{k}\right)\right)+\sigma_{0}\left(\delta B_{k}-\alpha Y_{k} \delta B_{k}^{\top} Y_{k}-\beta Y_{k} Y_{k}^{\top} \delta B_{k}\right) \\
& =-\delta_{k}\left(\nabla_{\mathcal{M}} \mathcal{F}\left(Y_{k}\right)\right)+\sigma_{0} P_{Y_{k}}\left(\delta B_{k}\right),
\end{aligned}
$$

and

(34)

$$
\begin{aligned}
A_{k}^{2} Y_{k} & =\left(Z_{k} Y_{k}^{\top}-Y_{k} Z_{k}^{\top}\right) A_{k} Y_{k} \\
& =\left[\delta_{k}\left(-G_{k} Y_{k}^{\top}+Y_{k} G_{k}^{\top}\right)+\sigma_{0}\left(\left(I-\beta Y_{k} Y_{k}^{\top}\right) \delta B_{k} Y_{k}^{\top}-Y_{k} \delta B_{k}^{\top}\left(I-\beta Y_{k} Y_{k}^{\top}\right)\right)\right] \\
& \cdot\left[-\delta_{k} \nabla_{\mathcal{M}} \mathcal{F}\left(Y_{k}\right)+\sigma_{0} P_{Y_{k}}\left(\delta B_{k}\right)\right] .
\end{aligned}
$$

We claim that

(35) $\mathbb{E}\left\{\left[\left(I-\beta Y_{k} Y_{k}^{\top}\right) \delta B_{k} Y_{k}^{\top}-Y_{k} \delta B_{k}^{\top}\left(I-\beta Y_{k} Y_{k}^{\top}\right)\right]\left(P_{Y_{k}}\left(\delta B_{k}\right)\right) \mid Y_{k}\right\}=-(n-1) \delta_{k} Y_{k}$. 
In fact, we can show that

$$
\begin{aligned}
& \mathbb{E}\left\{\left[\left(I-\beta Y_{k} Y_{k}^{\top}\right) \delta B_{k} Y_{k}^{\top}-Y_{k} \delta B_{k}^{\top}\left(I-\beta Y_{k} Y_{k}^{\top}\right)\right]\left(P_{Y_{k}}\left(\delta B_{k}\right)\right) \mid Y_{k}\right\} \\
= & \mathbb{E}\left\{\left(\delta B_{k} Y_{k}^{\top}-\beta Y_{k} Y_{k}^{\top} \delta B_{k} Y_{k}^{\top}-Y_{k} \delta B_{k}^{\top}+\beta Y_{k} \delta B_{k}^{\top} Y_{k} Y_{k}^{\top}\right)\right. \\
\cdot & \left.\left(\delta B_{k}-\alpha Y_{k} \delta B_{k}^{\top} Y_{k}-\beta Y_{k} Y_{k}^{\top} \delta B_{k}\right) \mid Y_{k}\right\} .
\end{aligned}
$$

Let $Q_{k}=\left[Y_{k}, Y_{k}^{\perp}\right]$ and $N_{k}=Q_{k}^{\top} \delta B_{k}$, and one can show that the entries of $N_{k}$ are independent $N\left(0, \delta_{k}\right)$ variables. By substituting $\delta B_{k}=Q_{k} N_{k}$ back into the above equation and expanding the corresponding terms, we have

$$
\begin{gathered}
\mathbb{E}\left\{Q_{k} N_{k} Y_{k}^{\top} Q_{k} N_{k} \mid Y_{k}\right\}=\mathbb{E}\left\{Q_{k} N_{k}\left[I_{p}, 0\right] N_{k} \mid Y_{k}\right\}=\delta_{k} Q_{k}\left(\begin{array}{c}
I_{p} \\
0
\end{array}\right)=\delta_{k} Y_{k}, \\
-\alpha \mathbb{E}\left\{Q_{k} N_{k} Y_{k}^{\top} Y_{k} N_{k}^{\top} Q_{k}^{\top} Y_{k} \mid Y_{k}\right\}=-\delta_{k} \alpha p Q_{k} I_{n} Q_{k}^{\top} Y_{k}=-\delta_{k} \alpha p Y_{k}, \\
-\beta \mathbb{E}\left\{Q_{k} N_{k} Y_{k}^{\top} Y_{k} Y_{k}^{\top} Q_{k} N_{k} \mid Y_{k}\right\}=-\beta \mathbb{E}\left\{Q_{k} N_{k} I_{p}\left[I_{p}, 0\right] N_{k} \mid Y_{k}\right\}=-\delta_{k} \beta Y_{k}, \\
-\beta \mathbb{E}\left\{Y_{k} Y_{k}^{\top} Q_{k} N_{k} Y_{k}^{\top} Q_{k} N_{k} \mid Y_{k}\right\}=-\beta \mathbb{E}\left\{Y_{k}\left[I_{p}, 0\right] N_{k}\left[I_{p}, 0\right] N_{k} \mid Y_{k}\right\}=-\delta_{k} \beta Y_{k}, \\
\alpha \beta \mathbb{E}\left\{Y_{k} Y_{k}^{\top} Q_{k} N_{k} Y_{k}^{\top} Y_{k} N_{k}^{\top} Q_{k}^{\top} Y_{k} \mid Y_{k}\right\}=\alpha \beta \mathbb{E}\left\{Y_{k}\left[I_{p}, 0\right] N_{k} N_{k}^{\top}\left(\begin{array}{c}
I_{p} \\
0
\end{array}\right) \mid Y_{k}\right\}=\delta_{k} \alpha \beta p Y_{k}, \\
\beta^{2} \mathbb{E}\left\{Y_{k} Y_{k}^{\top} Q_{k} N_{k} Y_{k}^{\top} Y_{k} Y_{k}^{\top} Q_{k} N_{k} \mid Y_{k}\right\}=\beta^{2} \mathbb{E}\left\{Y_{k}\left[I_{p}, 0\right] N_{k}\left[I_{p}, 0\right] N_{k} \mid Y_{k}\right\}=\delta_{k} \beta^{2} Y_{k}, \\
-\mathbb{E}\left\{Y_{k} N_{k}^{\top} Q_{k}^{\top} Q_{k} N_{k} \mid Y_{k}\right\}=-\delta_{k} n Y_{k}, \\
\alpha \mathbb{E}\left\{Y_{k} N_{k}^{\top} Q_{k}^{\top} Y_{k} N_{k}^{\top} Q_{k}^{\top} Y_{k} \mid Y_{k}\right\}=\alpha \mathbb{E}\left\{Y_{k} N_{k}^{\top}\left(\begin{array}{c}
I_{p} \\
0
\end{array}\right) N_{k}^{\top}\left(\begin{array}{c}
I_{p} \\
0
\end{array}\right) \mid Y_{k}\right\}=\delta_{k} \alpha Y_{k}, \\
\beta \mathbb{E}\left\{Y_{k} N_{k}^{\top} Q_{k}^{\top} Y_{k} Y_{k}^{\top} Q_{k} N_{k} \mid Y_{k}\right\}=\beta \mathbb{E}\left\{Y_{k} N_{k}^{\top} \operatorname{diag}\left\{I_{p}, 0_{n-p}\right\} N_{k} \mid Y_{k}\right\}=\delta_{k} \beta p Y_{k}, \quad \text { (twice),} \\
-\alpha \beta \mathbb{E}\left\{Y_{k} N_{k}^{\top} Q_{k}^{\top} Y_{k} Y_{k}^{\top} Y_{k} N_{k}^{\top} Q_{k}^{\top} Y_{k} \mid Y_{k}\right\} \\
=-\alpha \beta \mathbb{E}\left\{Y_{k} N_{k}^{\top}\left(\begin{array}{c}
I_{p} \\
0
\end{array}\right) N_{k}^{\top}\left(\begin{array}{c}
I_{p} \\
0
\end{array}\right) \mid Y_{k}\right\}=-\delta_{k} \alpha \beta Y_{k}, \\
-\beta^{2} \mathbb{E}\left\{Y_{k} N_{k}^{\top} Q_{k}^{\top} Y_{k} Y_{k}^{\top} Y_{k} Y_{k}^{\top} Q_{k} N_{k} \mid Y_{k}\right\} \\
=-\beta^{2} \mathbb{E}\left\{Y_{k} N_{k}^{\top} \operatorname{diag}\left\{I_{p}, 0_{n-p}\right\} N_{k} \mid Y_{k}\right\}=-\delta_{k} \beta^{2} p Y_{k} .
\end{gathered}
$$

Taking sum of the above terms yields (35). A direct corollary of (35) is

$$
\mathbb{E}\left\|\mathbb{E}\left(Y_{k+1}-Y_{k} \mid Y_{k}\right)-\delta_{k}\left(-\nabla_{\mathcal{M}} \mathcal{F}\left(Y_{k}\right)-\frac{n-1}{2} \sigma_{0}^{2} Y_{k}\right)\right\|_{F}^{2} \leq C_{1}^{\prime} \delta_{k}^{3} .
$$

Hence, we can derive an estimation of the first term in (31) as $(38)$

$$
\begin{aligned}
& \mathbb{E}\left\|\sum_{k=0}^{k_{s}-1}\left[\mathbb{E}\left(Y_{k+1}-Y_{k} \mid Y_{k}\right)-\delta_{k}\left(-\nabla_{\mathcal{M}} \mathcal{F}\left(Y_{k}\right)-\frac{n-1}{2} \sigma_{0}^{2} Y_{k}\right)\right]\right\|_{F}^{2} \\
\leq & \delta\left(\sum_{k=0}^{k_{s}-1} \delta_{k}\right) \sum_{k=0}^{k_{s}-1} \frac{1}{\delta_{k}^{2}} \mathbb{E}\left\|\mathbb{E}\left(Y_{k+1}-Y_{k} \mid Y_{k}\right)-\delta_{k}\left(-\nabla_{\mathcal{M}} \mathcal{F}\left(Y_{k}\right)-\frac{n-1}{2} \sigma_{0}^{2} Y_{k}\right)\right\|_{F}^{2} \\
\leq & \delta\left(\sum_{k=0}^{k_{s}-1} \delta_{k}\right)\left(\sum_{k=0}^{k_{s}-1} C_{1}^{\prime} \delta_{k}\right) \leq C_{1} \delta .
\end{aligned}
$$


The first inequality of (38) is due to Cauchy-Schwartz inequality and $\delta=\max \left\{\delta_{k}\right\}$.

The second term of (31) can be evaluated in view that all the cross-product terms vanish under the Frobenius norm:

$$
\begin{aligned}
& \mathbb{E}\left\|\sum_{k=0}^{k_{s}-1}\left[Y_{k+1}-Y_{k}-\mathbb{E}\left(Y_{k+1}-Y_{k} \mid Y_{k}\right)-\sigma_{0} P_{Y_{k}}\left(\delta B_{k}\right)\right]\right\|_{F}^{2} \\
= & \sum_{k=0}^{k_{s}-1} \mathbb{E}\left\|\left[Y_{k+1}-Y_{k}-\mathbb{E}\left(Y_{k+1}-Y_{k} \mid Y_{k}\right)-\sigma_{0} P_{Y_{k}}\left(\delta B_{k}\right)\right]\right\|_{F}^{2} \\
\leq & \sum_{k=0}^{k_{s}-1}\left(\mathbb{E}\left\|A_{k} Y_{k}-\sigma_{0} P_{Y_{k}}\left(\delta B_{k}\right)\right\|_{F}^{2}+C_{2}^{\prime} \delta_{k}^{2}\right) \\
\leq & \sum_{k=0}^{k_{s}-1} C_{2} \delta_{k}^{2} \leq C_{2} \delta .
\end{aligned}
$$

The third term of (31) can be estimated in view of the smoothness of $\nabla_{\mathcal{M}} \mathcal{F}$ :

$$
\begin{aligned}
& \mathbb{E}\left\|\sum_{k=0}^{k_{s}-1}\left[\int_{\tau_{k}}^{\tau_{k+1}}\left[-\nabla_{\mathcal{M}} \mathcal{F}(X(\tau))\right] \mathrm{d} \tau+\delta_{k} \nabla_{\mathcal{M}} \mathcal{F}\left(Y_{k_{\tau}}\right)\right]\right\|_{F}^{2} \\
= & \mathbb{E}\left\|\int_{0}^{\tau_{k_{s}}}\left[\nabla_{\mathcal{M}} \mathcal{F}\left(Y_{k_{\tau}}\right)-\nabla_{\mathcal{M}} \mathcal{F}(X(\tau))\right] \mathrm{d} \tau\right\|_{F}^{2} \\
\leq & T \int_{0}^{\tau_{k_{s}}} \mathbb{E}\left\|\left[\nabla_{\mathcal{M}} \mathcal{F}\left(Y_{k_{\tau}}\right)-\nabla_{\mathcal{M}} \mathcal{F}(X(\tau))\right]\right\|_{F}^{2} \mathrm{~d} \tau \\
\leq & T C_{3}^{\prime} \int_{0}^{\tau_{k_{s}}} \mathbb{E}\left\|X(\tau)-Y_{k_{\tau}}\right\|_{F}^{2} \mathrm{~d} \tau \\
= & T C_{3}^{\prime} \int_{0}^{\tau_{k_{s}}} R(\tau) \mathrm{d} \tau \leq T C_{3}^{\prime} \int_{0}^{t} R(\tau) \mathrm{d} \tau:=C_{3} \int_{0}^{t} R(\tau) \mathrm{d} \tau .
\end{aligned}
$$

Similarly one can show that the forth term of (31) can be bounded by

$$
\mathbb{E}\left\|\sum_{k=0}^{k_{s}-1}\left[\int_{\tau_{k}}^{\tau_{k+1}}\left(\frac{n-1}{2} \sigma_{0}^{2} X(\tau)\right) \mathrm{d} \tau-\delta_{k}\left(\frac{n-1}{2} \sigma_{0}^{2} Y_{k_{\tau}}\right)\right]\right\|_{F}^{2} \leq C_{4} \int_{0}^{t} R(\tau) \mathrm{d} \tau
$$

The fifth term of (31) can be estimated using Ito's isometry and the smoothness of $P_{u v}$ :

$$
\begin{aligned}
& \mathbb{E}\left\|\sigma_{0} \sum_{k=0}^{k_{s}-1}\left[\int_{\tau_{k}}^{\tau_{k+1}} P_{X(\tau)}(\mathrm{d} B(\tau))-P_{Y_{k}}\left(\delta B_{k}\right)\right]\right\|_{F}^{2} \\
= & \sigma_{0}^{2} \mathbb{E} \| \sum_{u, v} \int_{0}^{\tau_{k_{s}}}\left[P_{u v}\left(X(\tau)-P_{u v}\left(Y_{k_{\tau}}\right)\right] \mathrm{d} B_{u v}(\tau) \|_{F}^{2}\right. \\
= & \sigma_{0}^{2} \sum_{u, v} \int_{0}^{\tau_{k_{s}}} \mathbb{E}\left\|P_{u v}\left(X_{\tau}\right)-P_{u v}\left(Y_{k_{\tau}}\right)\right\|_{F}^{2} \mathrm{~d} \tau \\
\leq & C_{5} \int_{0}^{\tau_{k_{s}}} \mathbb{E}\left\|X(\tau)-Y_{k_{\tau}}\right\|_{F}^{2} \mathrm{~d} \tau=C_{5} \int_{0}^{\tau_{k_{s}}} R(\tau) \mathrm{d} \tau \leq C_{5} \int_{0}^{t} R(\tau) \mathrm{d} \tau .
\end{aligned}
$$


The last two terms can be estimated as

$$
\mathbb{E}\left\|\int_{\tau_{k_{s}}}^{s}\left(-\nabla_{\mathcal{M}} \mathcal{F}(X(\tau))-\frac{n-1}{2} \sigma_{0}^{2} X(\tau)\right) \mathrm{d} \tau\right\|_{F}^{2} \leq C_{6} \delta^{2} .
$$

and

$$
\mathbb{E}\left\|\sigma_{0} \int_{\tau_{k_{s}}}^{s} P_{X(\tau)}(\mathrm{d} B(\tau))\right\|_{F}^{2} \leq C_{7} \delta .
$$

Taking the above estimation together yields

$$
R(t) \leq 7\left[\left(C_{1}+C_{2}+C_{7}\right) \delta+C_{6} \delta^{2}+\left(C_{3}+C_{4}+C_{5}\right) \int_{0}^{t} R(\tau) \mathrm{d} \tau\right] .
$$

It follows directly from the Gronwall inequality that

$$
R(t) \leq C \delta, \quad \delta \in\left(0, \delta_{0}\right),
$$

where $C$ is A constant independent of $\delta$ and $\delta_{0}>0$.

4. IDDM Algorithm and Global convergence Analysis. Now we can generalize existing methods based on diffusion equation (9) to the Stiefel Manifold. The method we use in the following is a generalization of Intermittent Diffusion (ID) [7], namely Intermittent Diminishing Diffusion on Stiefel Manifold (IDDM), in which the diffusion strength is diminishing in every single cycle.

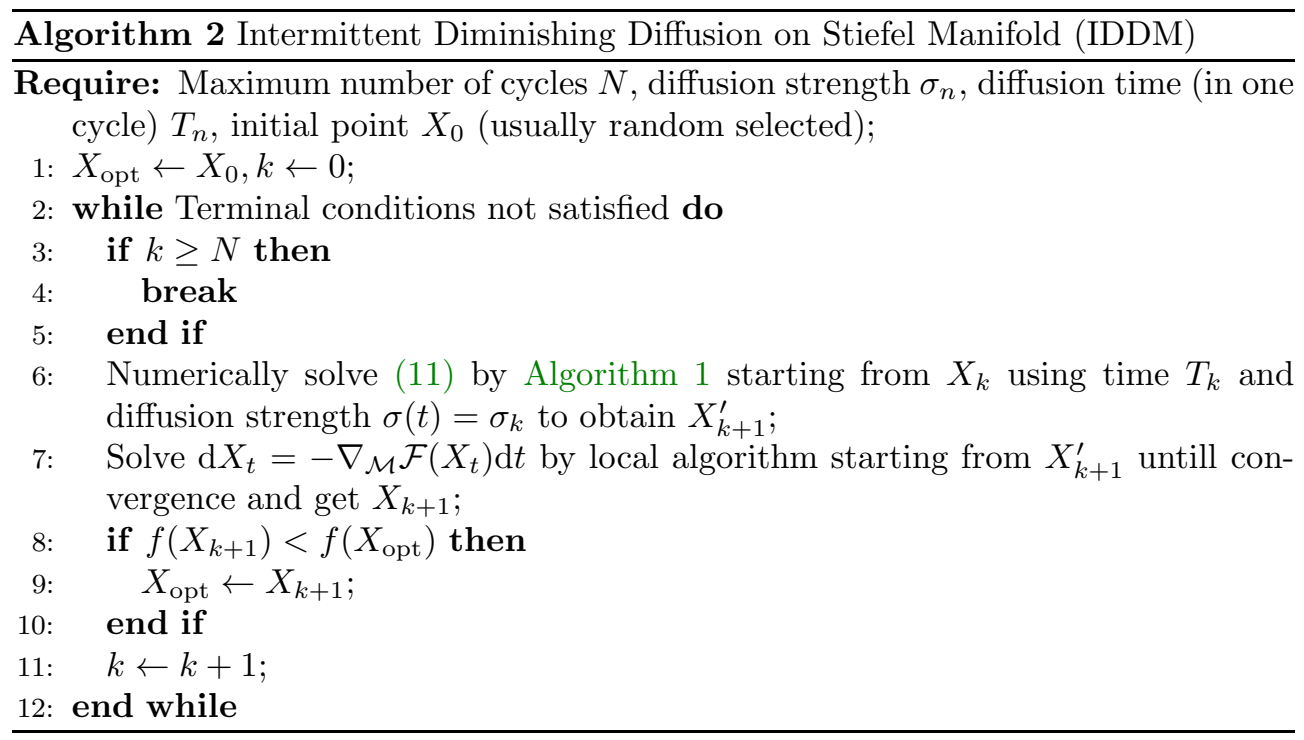

We first notice that the method can also be viewed as selecting

$$
\sigma(t)=\sum_{i=1}^{N} \sigma_{i} I_{\left[S_{i}, S_{i}+T_{i}\right]}(t),
$$

where $S_{i}$ is the starting time of each piece.

To provide the convergence results, we will first give some analysis for the FokkerPlanck Equation (25). The classic results yield the next theorem. 
Theorem 11. Assume that $\sigma(t)=\sigma_{0}$ is a constant. The distribution $p(x, t)$ converges $\left(\ell_{1}\right)$ to the Gibbs distribution

$$
\tilde{p}_{\sigma_{0}}(x)=\frac{1}{Z} e^{-2 \mathcal{F} / \sigma_{0}^{2}},
$$

where $Z$ is the normalization constant $Z=\int_{\mathcal{M}} e^{-2 \mathcal{F} / \sigma_{0}^{2}}$.

Proof. To simplify the problem, we set $\sigma_{0}=\sqrt{2}$ or let $t^{\prime} \leftarrow 2 t / \sigma_{0}^{2}$ and $\mathcal{F}^{\prime} \leftarrow 2 \mathcal{F} / \sigma_{0}^{2}$ to transfer the Fokker-Planck equation to one with $\sigma_{0}=\sqrt{2}$. Define the relative entropy

$$
H(p \mid q)=\int_{\mathcal{M}} p \log \left(\frac{p}{q}\right) \mathrm{d} x,
$$

for any two probability density function $p, q$ (on the manifold). The Csiszár-Kullback inequality shows that

$$
\|p-q\|_{\ell_{1}}^{2} \leq 2 H(p \mid q) .
$$

The canonical Stiefel manifold is knows as Einstein manifold in the case of $n=p$ and thus the Ricci curvature is positive definite [25].

For general canonical stiefel manifolds, the same results are shown in [13]. It is given in $[21,34]$ that $\mathcal{F}_{0}(X)=0$ satisfies a logarithmic Sobolev inequality with constant $\lambda_{0}$ which is the smallest eigenvalue of the Ricci curvature. It follows from $[21,34]$ that $\mathcal{F}(X)$ also satisfies a logarithmic Sobolev inequality with constant $\lambda=$ $\lambda_{0}\left(\max _{\mathcal{F}}-\min _{\mathcal{F}}\right)$, which indicates that

$$
\left.H\left(p(:, t) \mid \tilde{p}_{\sigma_{0}}\right) \leq e^{-2 \lambda t} H\left(p(:, 0) \mid \tilde{p}_{\sigma_{0}}\right)\right) .
$$

From the above analysis, we have

$$
\left\|p(:, t)-\tilde{p}_{\sigma_{0}}\right\|_{\ell_{1}}^{2} \leq 2 e^{-2 \lambda t} H\left(p(:, 0) \mid \tilde{p}_{\sigma_{0}}\right),
$$

which completes the proof.

We next provide the convergence results of Algorithm 2, which is nearly the same to the proof in [7] in the Euclidean space.

Theorem 12 (Convergence of Algorithm 2). Assume that the local algorithm satisfies $\mathcal{F}\left(X_{k}\right) \leq \mathcal{F}\left(X_{k}^{\prime}\right)$. Let the set of global minimizers be $P$, the global minimum be $\mathcal{F}^{*}$, and $X_{\text {opt }}$ to be the optimal solution obtained by Algorithm 2. For any given $\epsilon>0$ and $\zeta>0$, let $U$ be the basin of global minima, i.e., $U=\left\{X \in \mathcal{M}_{n, p} \mid \mathcal{F}(X)<\mathcal{F}^{*}+\zeta\right\}$. Then the following two statements hold:

1. $\forall \eta \in(0,1), \exists \sigma>0$ and $T>0$ (as a function of $\sigma$ ) such that if $\sigma_{i} \leq \sigma$ and $T_{i}>T\left(\sigma_{i}\right)$, then $\mathbb{P}\left(X_{i}^{\prime} \in U\right) \geq \eta$.

2. The probability to reach $U$ after $N$ cycles is at least $1-(1-\eta)^{N}$, namely, $\mathbb{P}\left(\exists i, X_{i}^{\prime} \in U\right)>1-(1-\eta)^{N}$. Thus, there exists $N_{0}>0$ such that if $\sigma_{i} \leq \sigma$, $T_{i}>T\left(\sigma_{i}\right)$ and $N>N_{0}$,

$$
\mathbb{P}\left(\mathcal{F}\left(X_{\text {opt }}\right)<\mathcal{F}^{*}+\zeta\right) \geq 1-\epsilon .
$$

Proof. A small neighborhood $U$ can be given so that $\forall X \in U, \mathcal{F}(X)<\mathcal{F}^{*}+\zeta$. We only need to prove that $\mathbb{P}\left(\exists k\right.$, s.t. $\left.X_{k}^{\prime} \in U\right) \geq 1-\epsilon$. 
From (48), $\forall \eta \in(0,1), \exists \sigma>0$ such that if $\sigma_{i} \leq \sigma$

$$
\int_{U} \tilde{p}_{\sigma_{i}}(x) \mathrm{d} x>\eta+(1-\eta) / 2 .
$$

Meanwhile, Theorem 11 yields that $\exists T>0$ such that if $T_{i}>T$,

$$
\left\|p\left(:, S_{i}+T_{i}\right)-\tilde{p}_{\sigma_{i}}\right\|_{\ell_{1}}<(1-\eta) / 2 .
$$

Hence, we have

$$
\mathbb{P}\left(X_{i}^{\prime} \in U\right)=\int_{U} \tilde{p}_{\sigma_{i}} d x-\int_{U} \tilde{p}_{\sigma_{i}}-p\left(x, S_{i}+T_{i}\right) d x \geq \eta .
$$

Independent intervals yields that

$$
\mathbb{P}\left(\forall i, X_{i}^{\prime} \in U^{c}\right)<(1-\eta)^{N} .
$$

Select a proper $N_{0}$ such that $(1-\eta)^{N_{0}} \leq \epsilon$ and we complete the proof.

REMARK 13. The results of Theorem 12 can be improved if we impose some stronger conditions on the object function and the local algorithm. If 1) the local algorithm always achieve a nearest local minimizer and 2) there are finite local minimizers (which is acceptable for a compact set), then the results can be improved as $\mathbb{P}\left(\operatorname{dist}\left(X_{\text {opt }}, P\right)<\zeta\right) \geq 1-\epsilon$. The proof is the same as [7].

REMARK 14. We have provided some analysis for the piecewise constant $\sigma(t)$ proposed by [7]. Notice that other $\sigma(t)$ may also give global convergence. For example, one can apply the $\sigma(t)=c / \sqrt{\log (t+2)}$ given by CDD and the proof of convergence is the same. One can refer to $[6,9]$ for the proof.

5. Numerical Experiments. In this section, we demonstrate the effectiveness of IDD methods on Stiefel Manifold (IDDM) on a variety of test problems. The first two subsections are devoted to the spherically constrained problems, while the last one focuses on the orthogonality constrained problem. We should point out that we have also test many problems, such as conformal mapping [12, 15], p-Harmonic flow [17, 32, 33, 11], compressed modes [26] and nonlinear eigenvalue problem in density functional theory $[19,35]$. They are not choosen in this section because the local algorithm is often able to return a pretty solution (or even "global solution") in a single run.

The performance of IDDM is mainly compared with the Random-Start local method dubbed as RSlocal, which randomly selects an initial point and then performs the local algorithm. The local algorithm that we employ is the curvilinear search method with Barzilai-Borwein steps (Algorithm 2 in [36]). Each run of IDDM consists of ten cycles while RSlocal is made up of ten trials of the local algorithm starting from randomly generated points. The parameter $\sigma_{i}$ in (47) is set to $\sigma_{i}=\alpha /\left(i d_{t}\right)^{1 / 2(n-1)}$, where $d_{t}$ is the step length, $n$ is the dimension of the variables and $\alpha$ is the initial diffusion strength. All experiments were performed on a workstation with an Intel Xeon E5-2640 v3 2.60GHz processor with access to $64 \mathrm{~GB}$ of RAM.

5.1. Homogeneous Polynomial Optimization. In this subsection, we evaluate the performance on homogeneous polynomial problems. The test polynomial is selected from [36] which cannot be globally minimized effectively by the local methods:

$$
\min _{x \in \mathbb{R}^{n}} \mathcal{F}(x)=\sum_{1 \leq i \leq n} x_{i}^{6}+\sum_{1 \leq i \leq n-1} x_{i}^{3} x_{i+1}^{3}, \quad \text { s.t. } \quad\|x\|_{2}=1 .
$$


For each of $n=10,20, \ldots, 200$, we repeat 50 independent runs of IDDM and RSlocal. The initial diffusion strength $\alpha$ is selected as $1 / n$. The minimum, mean and maximum of the objective function values, as well as the averaged cpu time in seconds are reported in Table 1. The corresponding mean and min are further illustrated in the left side of Figure 1. Our numerical results indicate that IDDM are always much better than RSlocal in this problem.

We further numerically explore the dependency of the performance of IDDM to the diffusion strength $\alpha$. For each $n$ ranging from 40 to 200, we repeat 50 independent tests of RSlocal and denote the averaged objective function values as $\mathcal{F}_{\text {RSlocal }}$. Similarly, we repeat 50 runs of IDDM with different initial diffusion strengths $\sigma$ from $10^{-4}$ to $10^{0}$ and the averaged objective function values are denoted by $\mathcal{F}_{\text {IDDM }}$. Each pixel in the right side of Figure 1 is a value of $-\log _{10}\left(\mathcal{F}_{\text {IDDM }} / \mathcal{F}_{\text {RSlocal }}\right)$. A positive value indicates an improvement achieved by IDDM over RSlocal while a negative value means that IDDM is worse than RSlocal. This image clearly shows that our IDDM outperforms RSlocal with the right choice of the diffusion strength illustrated in the region with the red color.

TABLE 1

Numerical results of polynomial optimization (58)

\begin{tabular}{|c|ccc|c|ccc|c|}
\hline \multirow{2}{*}{$n$} & \multicolumn{4}{|c|}{ RSlocal } & \multicolumn{4}{c|}{ IDDM } \\
\cline { 2 - 9 } & min & mean & max & cpu $(\mathrm{s})$ & min & mean & max & cpu $(\mathrm{s})$ \\
\hline 10 & $7.2 \mathrm{e}-04$ & $2.5 \mathrm{e}-03$ & $6.3 \mathrm{e}-03$ & 0.041 & $7.2 \mathrm{e}-04$ & $2.5 \mathrm{e}-03$ & $4.8 \mathrm{e}-03$ & 0.044 \\
\hline 20 & $1.1 \mathrm{e}-04$ & $6.1 \mathrm{e}-04$ & $1.3 \mathrm{e}-03$ & 0.047 & $5.0 \mathrm{e}-05$ & $3.3 \mathrm{e}-04$ & $1.1 \mathrm{e}-03$ & 0.087 \\
\hline 30 & $7.6 \mathrm{e}-05$ & $2.8 \mathrm{e}-04$ & $4.8 \mathrm{e}-04$ & 0.057 & $1.5 \mathrm{e}-05$ & $1.1 \mathrm{e}-04$ & $2.5 \mathrm{e}-04$ & 0.116 \\
\hline 40 & $7.5 \mathrm{e}-05$ & $1.6 \mathrm{e}-04$ & $2.5 \mathrm{e}-04$ & 0.065 & $5.4 \mathrm{e}-06$ & $4.5 \mathrm{e}-05$ & $1.2 \mathrm{e}-04$ & 0.129 \\
\hline 50 & $5.4 \mathrm{e}-05$ & $1.1 \mathrm{e}-04$ & $1.7 \mathrm{e}-04$ & 0.078 & $2.2 \mathrm{e}-06$ & $2.1 \mathrm{e}-05$ & $5.5 \mathrm{e}-05$ & 0.166 \\
\hline 60 & $2.7 \mathrm{e}-05$ & $8.0 \mathrm{e}-05$ & $1.4 \mathrm{e}-04$ & 0.087 & $2.1 \mathrm{e}-06$ & $1.3 \mathrm{e}-05$ & $3.5 \mathrm{e}-05$ & 0.154 \\
\hline 70 & $2.9 \mathrm{e}-05$ & $6.0 \mathrm{e}-05$ & $9.0 \mathrm{e}-05$ & 0.100 & $1.0 \mathrm{e}-06$ & $9.9 \mathrm{e}-06$ & $2.6 \mathrm{e}-05$ & 0.173 \\
\hline 80 & $2.0 \mathrm{e}-05$ & $4.5 \mathrm{e}-05$ & $6.3 \mathrm{e}-05$ & 0.095 & $1.3 \mathrm{e}-06$ & $8.1 \mathrm{e}-06$ & $4.2 \mathrm{e}-05$ & 0.153 \\
\hline 90 & $2.3 \mathrm{e}-05$ & $3.7 \mathrm{e}-05$ & $5.3 \mathrm{e}-05$ & 0.099 & $1.2 \mathrm{e}-06$ & $5.4 \mathrm{e}-06$ & $1.7 \mathrm{e}-05$ & 0.169 \\
\hline 100 & $1.7 \mathrm{e}-05$ & $3.1 \mathrm{e}-05$ & $4.5 \mathrm{e}-05$ & 0.104 & $6.7 \mathrm{e}-07$ & $4.3 \mathrm{e}-06$ & $1.4 \mathrm{e}-05$ & 0.175 \\
\hline 110 & $1.6 \mathrm{e}-05$ & $2.6 \mathrm{e}-05$ & $3.6 \mathrm{e}-05$ & 0.118 & $4.6 \mathrm{e}-07$ & $3.0 \mathrm{e}-06$ & $8.6 \mathrm{e}-06$ & 0.166 \\
\hline 120 & $1.5 \mathrm{e}-05$ & $2.3 \mathrm{e}-05$ & $3.1 \mathrm{e}-05$ & 0.115 & $6.2 \mathrm{e}-07$ & $2.6 \mathrm{e}-06$ & $8.9 \mathrm{e}-06$ & 0.174 \\
\hline 130 & $8.3 \mathrm{e}-06$ & $1.9 \mathrm{e}-05$ & $2.5 \mathrm{e}-05$ & 0.125 & $3.2 \mathrm{e}-07$ & $2.3 \mathrm{e}-06$ & $4.6 \mathrm{e}-06$ & 0.191 \\
\hline 140 & $6.8 \mathrm{e}-06$ & $1.6 \mathrm{e}-05$ & $2.1 \mathrm{e}-05$ & 0.128 & $7.5 \mathrm{e}-07$ & $2.4 \mathrm{e}-06$ & $7.3 \mathrm{e}-06$ & 0.172 \\
\hline 150 & $9.9 \mathrm{e}-06$ & $1.5 \mathrm{e}-05$ & $2.1 \mathrm{e}-05$ & 0.136 & $6.7 \mathrm{e}-07$ & $2.0 \mathrm{e}-06$ & $6.9 \mathrm{e}-06$ & 0.180 \\
\hline 160 & $8.3 \mathrm{e}-06$ & $1.3 \mathrm{e}-05$ & $1.8 \mathrm{e}-05$ & 0.140 & $3.4 \mathrm{e}-07$ & $1.8 \mathrm{e}-06$ & $7.5 \mathrm{e}-06$ & 0.184 \\
\hline 170 & $7.9 \mathrm{e}-06$ & $1.1 \mathrm{e}-05$ & $1.4 \mathrm{e}-05$ & 0.153 & $3.2 \mathrm{e}-07$ & $1.9 \mathrm{e}-06$ & $5.0 \mathrm{e}-06$ & 0.183 \\
\hline 180 & $7.9 \mathrm{e}-06$ & $1.0 \mathrm{e}-05$ & $1.3 \mathrm{e}-05$ & 0.152 & $5.4 \mathrm{e}-07$ & $1.8 \mathrm{e}-06$ & $5.0 \mathrm{e}-06$ & 0.186 \\
\hline 190 & $7.3 \mathrm{e}-06$ & $9.5 \mathrm{e}-06$ & $1.2 \mathrm{e}-05$ & 0.154 & $4.1 \mathrm{e}-07$ & $1.7 \mathrm{e}-06$ & $3.4 \mathrm{e}-06$ & 0.190 \\
\hline 200 & $5.2 \mathrm{e}-06$ & $8.5 \mathrm{e}-06$ & $1.1 \mathrm{e}-05$ & 0.160 & $5.8 \mathrm{e}-07$ & $2.0 \mathrm{e}-06$ & $7.0 \mathrm{e}-06$ & 0.189 \\
\hline
\end{tabular}

5.2. Biquadratic optimization. We next consider the so-called biquadratic optimization over unit spheres [18]:

$$
\begin{array}{rl}
\min _{x \in \mathbb{R}^{n}, y \in \mathbb{R}^{n}} & b(x, y)=\sum_{1 \leq i, k \leq n, 1 \leq j, l \leq n} b_{i j k l} x_{i} y_{j} x_{k} y_{l} \\
\text { s.t. } & \|x\|=1,\|y\|=1 .
\end{array}
$$

Without loss of generality, we impose the symmetric property $b_{i j k l}=b_{k j i l}=b_{i l k j}$ for $i, k, j, l=1, \ldots, n$. A semidefinite programing relaxation approach is proposed in [18]. Since Examples 5.1 to 5.3 in this reference can be easily found by local solvers, we generate the coefficients $b_{i j k l}$ as following: 
FIG. 1. (a) The objective function values of IDDM an RSlocal on (58). (b) $-\log _{10}\left(\mathcal{F}_{\text {IDDM }} / \mathcal{F}_{\mathrm{RSlocal}}\right)$, i.e., the performance of IDDM using various initial diffusion strength with respect to RSlocal.

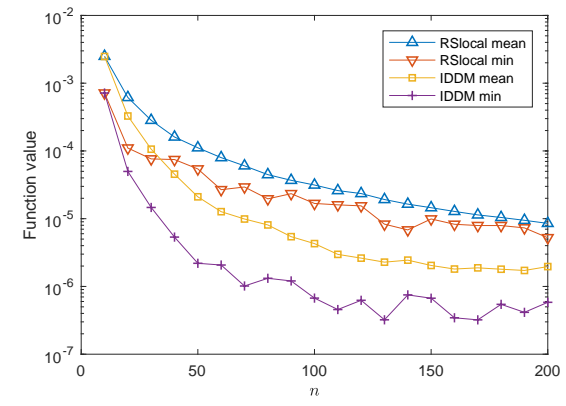

(a)

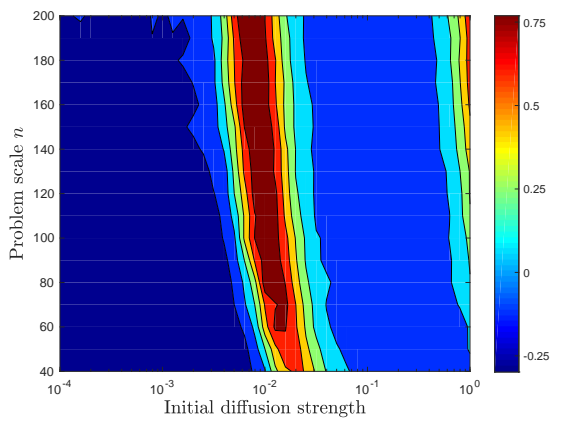

(b)

case i) $b_{i j k l}=(-1)^{i+j+k+l}|c|$, where $c$ is a Gaussian random variable.

case ii) $b_{i j k l}=\left|c_{1}\right| 1_{c_{2}>\eta}$, where $c_{1}$ is a Gaussian random variable, $c_{2}$ is uniformly distributed in $[0,1]$ and $\eta \in(0,1)$.

For each of $n=6,7, \ldots, 25$, we repeat 50 independent runs of IDDM and RSlocal. For the parameter $\alpha$ of IDDM, we select a few values in $\left[10^{-4}, 10^{2}\right]$ for each $n$ and choose the one with the best performance. The minimum, mean and maximum of the difference between the objective function values and the smallest objective function value identified in the 50 runs are reported in Tables 2 and 3. From the tables, we can see that both IDDM and RSlocal can find the "smallest" function values. IDDM usually performs better than RSlocal in most cases in terms of the mean value.

TABLE 2

Numerical results of biquadratic optimization: case $i$

\begin{tabular}{|c|cccc|cccc|}
\hline \multirow{2}{*}{$n$} & \multicolumn{4}{|c|}{ RSlocal } & \multicolumn{4}{c|}{ IDDM } \\
\cline { 2 - 9 } & min & mean & max & cpu $(\mathrm{s})$ & min & mean & max & cpu $(\mathrm{s})$ \\
\hline 6 & $5.2 \mathrm{e}-14$ & $1.4 \mathrm{e}-02$ & $2.5 \mathrm{e}-01$ & 0.033 & $1.5 \mathrm{e}-14$ & $8.3 \mathrm{e}-03$ & $1.8 \mathrm{e}-02$ & 0.034 \\
\hline 7 & $3.6 \mathrm{e}-14$ & $2.2 \mathrm{e}-02$ & $2.8 \mathrm{e}-01$ & 0.020 & $3.7 \mathrm{e}-14$ & $1.4 \mathrm{e}-02$ & $2.8 \mathrm{e}-01$ & 0.030 \\
\hline 8 & $4.8 \mathrm{e}-14$ & $1.1 \mathrm{e}-01$ & $1.6 \mathrm{e}+00$ & 0.026 & $2.7 \mathrm{e}-15$ & $2.5 \mathrm{e}-13$ & $7.1 \mathrm{e}-13$ & 0.030 \\
\hline 9 & $2.5 \mathrm{e}-14$ & $1.4 \mathrm{e}-01$ & $3.9 \mathrm{e}-01$ & 0.032 & $2.2 \mathrm{e}-14$ & $4.3 \mathrm{e}-12$ & $2.6 \mathrm{e}-11$ & 0.037 \\
\hline 10 & $3.3 \mathrm{e}-14$ & $1.1 \mathrm{e}-01$ & $1.2 \mathrm{e}+00$ & 0.041 & $7.1 \mathrm{e}-15$ & $3.4 \mathrm{e}-12$ & $1.9 \mathrm{e}-11$ & 0.046 \\
\hline 11 & $2.7 \mathrm{e}-14$ & $8.0 \mathrm{e}-02$ & $6.4 \mathrm{e}-01$ & 0.046 & $2.8 \mathrm{e}-14$ & $1.6 \mathrm{e}-11$ & $1.8 \mathrm{e}-10$ & 0.054 \\
\hline 12 & $9.9 \mathrm{e}-14$ & $3.5 \mathrm{e}-02$ & $2.1 \mathrm{e}-01$ & 0.056 & $1.4 \mathrm{e}-13$ & $1.9 \mathrm{e}-02$ & $1.6 \mathrm{e}-01$ & 0.075 \\
\hline 13 & $3.7 \mathrm{e}-14$ & $2.5 \mathrm{e}-01$ & $7.7 \mathrm{e}-01$ & 0.059 & $2.0 \mathrm{e}-14$ & $2.0 \mathrm{e}-01$ & $7.7 \mathrm{e}-01$ & 0.076 \\
\hline 14 & $1.1 \mathrm{e}-13$ & $2.4 \mathrm{e}-01$ & $1.1 \mathrm{e}+00$ & 0.073 & $8.2 \mathrm{e}-14$ & $1.4 \mathrm{e}-01$ & $9.0 \mathrm{e}-01$ & 0.102 \\
\hline 15 & $1.2 \mathrm{e}-13$ & $1.3 \mathrm{e}-01$ & $5.2 \mathrm{e}-01$ & 0.085 & $1.2 \mathrm{e}-13$ & $4.8 \mathrm{e}-12$ & $6.4 \mathrm{e}-11$ & 0.088 \\
\hline 16 & $5.0 \mathrm{e}-13$ & $5.2 \mathrm{e}-02$ & $4.6 \mathrm{e}-01$ & 0.110 & $2.4 \mathrm{e}-12$ & $2.7 \mathrm{e}-02$ & $3.1 \mathrm{e}-01$ & 0.163 \\
\hline 17 & $1.4 \mathrm{e}-14$ & $6.5 \mathrm{e}-01$ & $1.5 \mathrm{e}+00$ & 0.112 & $3.0 \mathrm{e}-13$ & $3.9 \mathrm{e}-12$ & $6.2 \mathrm{e}-11$ & 0.111 \\
\hline 18 & $2.0 \mathrm{e}-13$ & $1.9 \mathrm{e}-01$ & $9.5 \mathrm{e}-01$ & 0.136 & $6.6 \mathrm{e}-14$ & $6.1 \mathrm{e}-02$ & $4.6 \mathrm{e}-01$ & 0.187 \\
\hline 19 & $2.9 \mathrm{e}-13$ & $3.6 \mathrm{e}-01$ & $9.5 \mathrm{e}-01$ & 0.186 & $1.4 \mathrm{e}-14$ & $2.5 \mathrm{e}-12$ & $1.9 \mathrm{e}-11$ & 0.178 \\
\hline 20 & $4.1 \mathrm{e}-14$ & $4.0 \mathrm{e}-01$ & $1.3 \mathrm{e}+00$ & 0.225 & $2.8 \mathrm{e}-13$ & $2.3 \mathrm{e}-01$ & $9.8 \mathrm{e}-01$ & 0.335 \\
\hline 21 & $4.3 \mathrm{e}-14$ & $4.9 \mathrm{e}-01$ & $1.2 \mathrm{e}+00$ & 0.267 & $4.4 \mathrm{e}-14$ & $3.0 \mathrm{e}-01$ & $8.7 \mathrm{e}-01$ & 0.409 \\
\hline 22 & $3.3 \mathrm{e}-13$ & $4.2 \mathrm{e}-01$ & $1.0 \mathrm{e}+00$ & 0.324 & $4.4 \mathrm{e}-13$ & $2.9 \mathrm{e}-11$ & $1.4 \mathrm{e}-10$ & 0.472 \\
\hline 23 & $1.0 \mathrm{e}-13$ & $6.9 \mathrm{e}-01$ & $1.8 \mathrm{e}+00$ & 0.410 & $2.7 \mathrm{e}-13$ & $6.7 \mathrm{e}-12$ & $2.5 \mathrm{e}-11$ & 0.454 \\
\hline 24 & $1.2 \mathrm{e}-13$ & $4.7 \mathrm{e}-01$ & $1.1 \mathrm{e}+00$ & 0.484 & $1.2 \mathrm{e}-12$ & $3.4 \mathrm{e}-01$ & $9.9 \mathrm{e}-01$ & 0.711 \\
\hline 25 & $5.6 \mathrm{e}-13$ & $3.4 \mathrm{e}-01$ & $1.1 \mathrm{e}+00$ & 0.556 & $5.1 \mathrm{e}-13$ & $3.1 \mathrm{e}-01$ & $1.2 \mathrm{e}+00$ & 0.876 \\
\hline
\end{tabular}


We next demonstrate the performance of IDDM with respect to the initial diffusion strength $\alpha$. For each $n=\{18,20\}$, we repeat 50 independent tests of RSlocal. The averaged difference to global objective function values is plotted as the red line in Figure 2. Then we repeat 50 runs of IDDM with different initial diffusion strengths $\sigma$ from $10^{-4}$ to $10^{2}$. The averaged difference to global objective function values are depicted as the blue curve in Figure 2. We can see that our IDDM outperforms RSlocal if the diffusion strength is chosen suitably. Similar behavior can be observed on other dimensions of $n$.

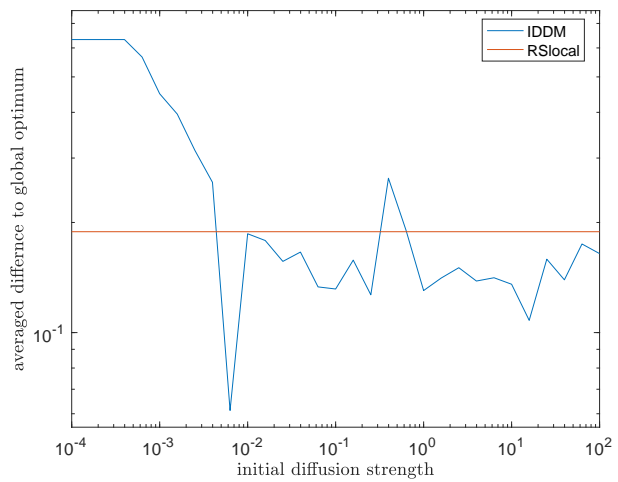

(a) $n=18$

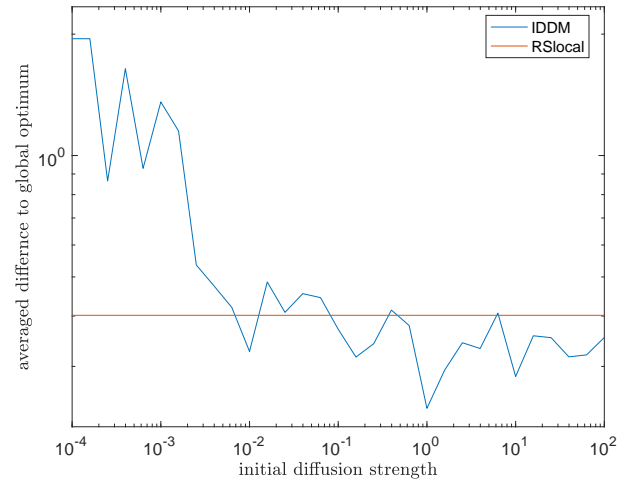

(b) $n=20$

FIG. 2. The performance of IDDM with respect to the initial diffusion strength

5.3. Computation of Stability Number. Let $G=(V, E)$ be an undirected graph. A stable (independent) set in $G$ is a set of vertices that are mutually nonadjacent. The stability number $S(G)$ for a given graph $G$ is defined as the size of a maximum stable set in $G$. It was shown by Motzkin and Straus [22] that

$$
S(G)^{-1}=\min _{\|x\|_{2}=1} \sum_{i=1}^{n} x_{i}^{4}+2 \sum_{(i, j) \in E} x_{i}^{2} x_{j}^{2},
$$

which is a single spherically constrainted problem. We select a few typical graphs as in [36] and we repeat 50 independent runs of IDDM and RSlocal. The parameter $\alpha$ is set to 0.005 in IDDM. The size $|V|$ of the graph, the mean and maximum of $S(G)$ as well as the cpu time are presented in Table 4. Note that the larger the value $S(G)$ is obtained, the better the stability number is estimated. We can see that IDDM almost always achieve a better solution than RSlocal.

5.4. Structure Determination in Cryo-EM. We now consider an example with multiple orthogonality constraints that arises from Cryo-EM [31]. In this test problem, we try to recover $N$ orientations $\left\{\tilde{R}_{i}\right\}$ from two dimensional (2D) projection images $\left\{P_{i}\right\}$ of a three dimensional (3D) object. Each $\tilde{R}_{i} \in \mathbb{R}^{3 \times 3}$ describes a $3 \mathrm{D}$ orthogonal matrix or rotation, i.e., $\tilde{R}_{i}^{\top} \tilde{R}_{i}=I_{3}$ and $\operatorname{det}\left(\tilde{R}_{i}\right)=1$. Let $\tilde{c}_{i j}=\left(x_{i j}, y_{i j}, 0\right)$ be the common line of the Fourier transforms of $P_{i}$ and $P_{j}$ (viewed in $P_{i}$ ). When the data are exact, it follows from the Fourier projection-slice theorem [31] that the common lines must coincide, i.e.,

$$
\tilde{R}_{i} \tilde{c}_{i j}=\tilde{R}_{j} \tilde{c}_{j i}
$$


Since the third column $\tilde{R}_{i}^{3}$ can be recovered from the first two columns $\tilde{R}_{i}^{1}$ and $\tilde{R}_{i}^{2}$ as $\tilde{R}_{i}^{3}= \pm \tilde{R}_{i}^{1} \times \tilde{R}_{i}^{2}$, the rotations $\left\{\tilde{R}_{i}\right\}$ can be compressed to $3 \times 2$ matrix. Therefore, the corresponding optimization problem can be formulated as

$$
\min _{R_{i}} \sum_{i=1}^{N} \rho\left(R_{i} c_{i j}, R_{j} c_{j i}\right), \quad \text { s.t. } \quad R_{i}^{\top} R_{i}=I_{2}, R_{i} \in \mathbb{R}^{3 \times 2}
$$

where $\rho$ is the function representing the distance between the two vectors, $R_{i}$ is made up of the first two columns of $\tilde{R}_{i}$ and $c_{i j}$ consists of the first two elements of $\tilde{c}_{i j}$. The distance function $\rho(u, v)=\|u-v\|_{2}$ is chosen in [31] and it leads to an eigenvector relaxation and semidfinite programming relxation. In our experiments, we select $\rho(u, v)=\|u-v\|_{q}$ with $q=0.5$ since it often leads to better mean square error defined as follows. Note that it holds $O \tilde{R}_{i} \tilde{c}_{i j}=O \tilde{R}_{j} \tilde{c}_{j i}$ for any fixed orthogonal matrix $O \in \mathbb{R}^{3 \times 3}$. Hence, we measure the error between the recovered rotations $\hat{R}_{i}$ and real rotations $\tilde{R}_{i}$ by the mean square error (MSE) defined as

$$
\mathrm{MSE}=\min _{O^{\top} O=I_{3}} \sum_{i=1}^{N}\left\|\hat{R}_{i}-O \tilde{R}_{i}\right\|_{F}^{2} .
$$

We compare IDDM with RSlocal and the eigenvector relaxation method developed in [31] (dubbed as "eigs"). The semidefinite programming relaxation approach in [31] is not compared because our experiments show that our local algorithm often can be better than it in terms of both accuracy and computational time. Each run of IDDM consists of ten cycles starting from the point generated from eigs while RSlocal is made up of ten trials of the local algorithm starting either from eigs or nine randomly generated points. The parameter $\alpha$ in IDDM is set to 0.1. In the subsequent experiments, "cpu" is the average cpu time of one cycle in seconds and "obj" stands for the final objective value.

Our first experiment is based on randomly generated data sets. We first create $N$ rotations $\tilde{R}_{i}$ by using the MATLAB command "orth( $(\operatorname{rand}(3,3))$ ". The common line vectors are computed next as $\tilde{c}_{i j}=\tilde{R}_{i}^{-1} \cdot\left(\tilde{R}_{i}^{3} \times \tilde{R}_{j}^{3}\right) /\left\|\tilde{R}_{i}^{3} \times \tilde{R}_{j}^{3}\right\|$ and $\tilde{c}_{j i}=\tilde{R}_{j}^{-1} \cdot\left(\tilde{R}_{j}^{3} \times \tilde{R}_{i}^{3}\right) /\left\|\tilde{R}_{j}^{3} \times \tilde{R}_{j}^{3}\right\|$ from each pair $\tilde{R}_{i}$ and $\tilde{R}_{j}$. After converting $\tilde{c}_{i j}$ and $\tilde{c}_{j i}$ into $c_{i j}$ and $c_{j i}$, we replace $c_{i j}$ and $c_{j i}$ by two random vectors that are sampled from the uniform distribution over the unit circle with probability $p$. That is, the common line vectors stay the same with probability $(1-p)$. We test the cases of $N=100,500,1000$. The computed objective function values are presented in the left column of Figure 3. The lines "eigs", "IDDM mean" and "IDDM min" are the objective function value computed by eigs, the averaged and minimum objective function value computed by IDDM, respectively. The lines "RSlocal mean" and "RSlocal min" are the corresponding values of RSlocal. We can see that both RSlocal and IDDM can find better objective function values than eigs. We should point that both RSlocal and IDDM can find the same minumum when they start from the initial point generated by eigs. However, IDDM performs better than RSlocal on average.

A detailed summary of the computational results are reported in Table 5. We further denote "mse1" as the smallest MSE generated in the ten cycles and "obj1" is the corresponding objective function value. Similarly, "obj2" stands the smallest objective value in ten cycles and the corresponding MSE is denoted as "mse2". We can see that the pairs "(mse1, obj1)" and "(mse2, obj2)" are almost the same except the last row of each of $N=100,500,1000$. The reason is that the initial point produced 
by eigs lies in a small neighbourhood of the global solution and our local algorithm starting from eigs usually can find this global solution successfully. Although other cycles can also identify a local solution, the corresponding objective funtion values are larger. For the cases that mse1 is different from mse2, it means that a smaller objective function value does not necessary have a smaller MSE in the noisy cases. The reason is that the model (60) does not characterize the original Cryo-EM problem well.

Our second experiment is based on the dataset from [31]. The noise-to-signal ratio (NSR) is defined as NSR $=\operatorname{Var}($ Noise $) / \operatorname{Var}($ Signal), where Signal is the clean projection image and Noise is the noise realization. The set up of the experiments is the same as the random data sets. The objective function values are plotted in the right column of Figure 3. They show that eigs itself can provide a good solution when NSR is small. The averaged objective function values obtained from IDDM are the best when NSR is larger. IDDM also can find a smaller objective function value in a few cases. The detailed summary of computational results are presented in Table 6. The pairs "(mse1, obj1)" and "(mse2, obj2)" are almost the same when NSR is small. However, for a large NSR, IDDM often is able to identify a smaller objective function value whose corresponding MSE is not the best. This observation again is not a contradiction but due to that the model (60) is not suitable in these cases. Nevertheless, these experiments are still perfect to show that IDDM is often better than the local algorithm itself and the local algorithm starting from multiple randomly generated initial points when the global solution is difficult to be captured.

6. Conclusion. The goal of this paper is to construct an algorithm which is able to identify global solutions of minimization with orthogonality constraints. Our strategy is simply alternating between a local algorithm on Stiefel manifold and a gradient flow method with stochastic diffusion on manifold. The main concept is that a suitable diffusion term is able to drive the iteration to escape the region around a local solution. We derive an extrinsic form of the Brownian motion on the manifold and design a numerical efficient scheme to solve the corresponding SDE on manifold. We further theoretically show the half order convergence of the proposed numerical method for solving SDE on the Stefiel manifold. Moreover, convergence to the global minimizer is also theoretically established as long as the diffusion is sufficiently enough. However, our extensive numerical experiments on polynomial optimization and 3D structure determination from Cryo-EM show that a few cycles of our algorithm is often able to provide a better solution than the local algorithm. Although both theoretical and numerical results are still limited in certain senses, they are indeed promising especially for problems with good structures. Our future work includes a better theoretical understanding the algorithms, refining them for more typical applications and some better ways on choosing or even learning the diffusion parameter $\sigma(t)$. 

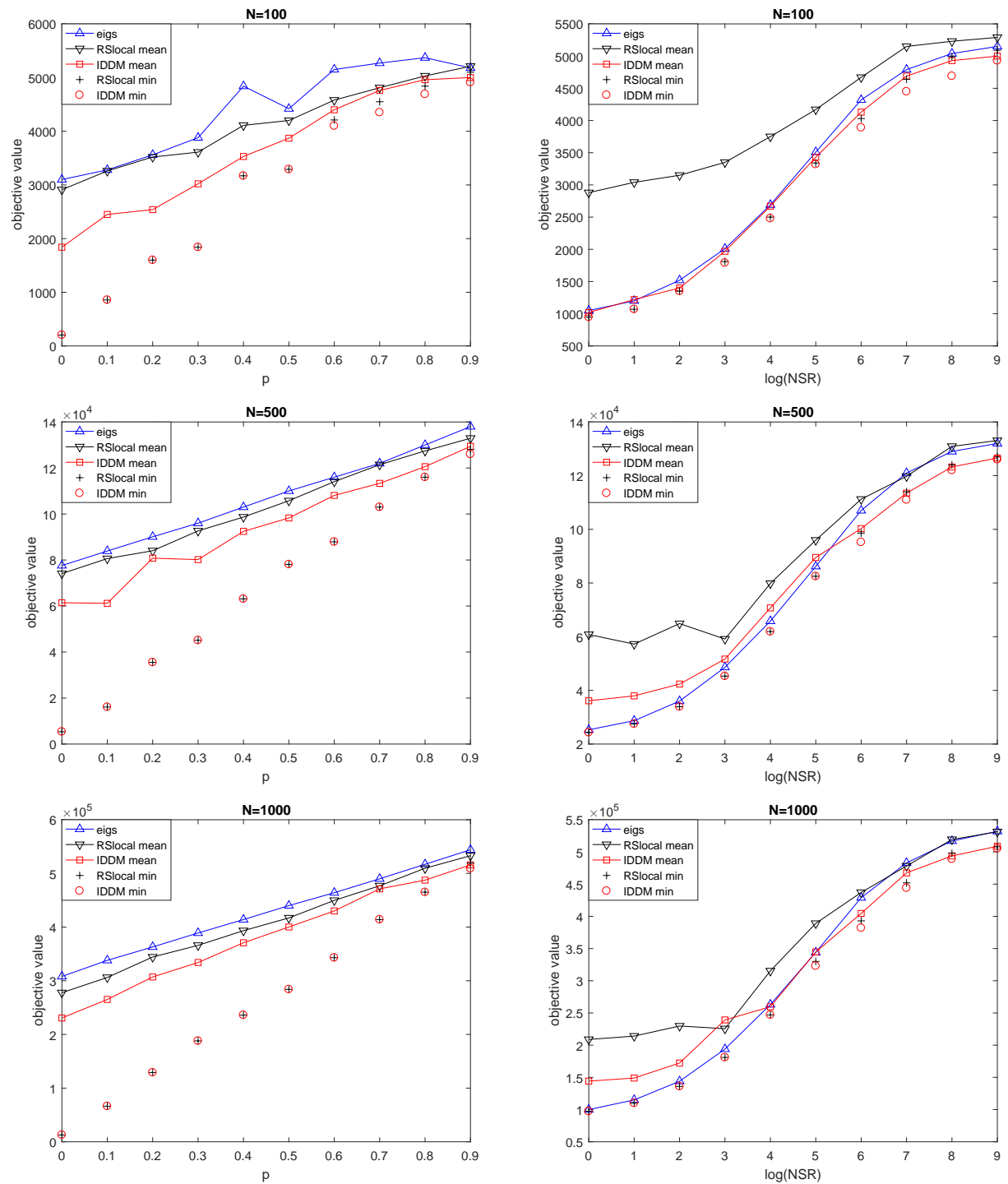

FIG. 3. The objective values for the random datasets (left column) and the dataset from [31] (right column) 
TABLE 3

Numerical results of biquadratic optimization: case ii

\begin{tabular}{|c|cccc|cccc|}
\hline \multirow{2}{*}{$n$} & \multicolumn{5}{|c|}{ RSlocal } & \multicolumn{4}{c|}{ IDDM } \\
\cline { 2 - 9 } & min & mean & max & cpu & min & mean & max & cpu \\
\hline 6 & $4.0 \mathrm{e}-14$ & $3.6 \mathrm{e}-04$ & $5.9 \mathrm{e}-03$ & 0.014 & $4.7 \mathrm{e}-14$ & $3.6 \mathrm{e}-04$ & $5.9 \mathrm{e}-03$ & 0.020 \\
\hline 7 & $3.2 \mathrm{e}-14$ & $7.1 \mathrm{e}-03$ & $1.2 \mathrm{e}-01$ & 0.016 & $4.2 \mathrm{e}-14$ & $1.1 \mathrm{e}-13$ & $2.8 \mathrm{e}-13$ & 0.016 \\
\hline 8 & $0.0 \mathrm{e}+00$ & $8.3 \mathrm{e}-03$ & $8.8 \mathrm{e}-02$ & 0.032 & $4.9 \mathrm{e}-15$ & $2.4 \mathrm{e}-03$ & $4.0 \mathrm{e}-02$ & 0.042 \\
\hline 9 & $5.6 \mathrm{e}-14$ & $7.0 \mathrm{e}-02$ & $4.2 \mathrm{e}-01$ & 0.020 & $2.3 \mathrm{e}-14$ & $2.2 \mathrm{e}-02$ & $4.2 \mathrm{e}-01$ & 0.028 \\
\hline 10 & $2.0 \mathrm{e}-14$ & $6.7 \mathrm{e}-02$ & $2.4 \mathrm{e}-01$ & 0.029 & $1.4 \mathrm{e}-14$ & $3.5 \mathrm{e}-02$ & $1.8 \mathrm{e}-01$ & 0.040 \\
\hline 11 & $9.5 \mathrm{e}-14$ & $7.4 \mathrm{e}-03$ & $7.1 \mathrm{e}-02$ & 0.031 & $6.8 \mathrm{e}-14$ & $2.4 \mathrm{e}-03$ & $6.3 \mathrm{e}-03$ & 0.046 \\
\hline 12 & $1.2 \mathrm{e}-13$ & $4.6 \mathrm{e}-02$ & $2.3 \mathrm{e}-01$ & 0.036 & $4.4 \mathrm{e}-14$ & $2.0 \mathrm{e}-12$ & $2.0 \mathrm{e}-11$ & 0.045 \\
\hline 13 & $1.4 \mathrm{e}-13$ & $5.2 \mathrm{e}-02$ & $2.7 \mathrm{e}-01$ & 0.039 & $4.3 \mathrm{e}-14$ & $2.5 \mathrm{e}-02$ & $2.9 \mathrm{e}-01$ & 0.061 \\
\hline 14 & $5.7 \mathrm{e}-14$ & $2.9 \mathrm{e}-01$ & $8.2 \mathrm{e}-01$ & 0.056 & $3.5 \mathrm{e}-14$ & $1.0 \mathrm{e}-01$ & $7.1 \mathrm{e}-01$ & 0.084 \\
\hline 15 & $7.1 \mathrm{e}-15$ & $6.0 \mathrm{e}-02$ & $2.7 \mathrm{e}-01$ & 0.064 & $3.8 \mathrm{e}-14$ & $2.7 \mathrm{e}-02$ & $3.0 \mathrm{e}-01$ & 0.092 \\
\hline 16 & $3.6 \mathrm{e}-14$ & $1.3 \mathrm{e}-01$ & $9.2 \mathrm{e}-01$ & 0.091 & $5.0 \mathrm{e}-14$ & $2.0 \mathrm{e}-12$ & $3.1 \mathrm{e}-12$ & 0.125 \\
\hline 17 & $3.5 \mathrm{e}-14$ & $5.6 \mathrm{e}-02$ & $7.8 \mathrm{e}-01$ & 0.091 & $1.3 \mathrm{e}-14$ & $5.1 \mathrm{e}-13$ & $7.6 \mathrm{e}-12$ & 0.141 \\
\hline 18 & $2.3 \mathrm{e}-13$ & $2.8 \mathrm{e}-01$ & $8.0 \mathrm{e}-01$ & 0.118 & $5.4 \mathrm{e}-14$ & $8.9 \mathrm{e}-14$ & $1.5 \mathrm{e}-13$ & 0.165 \\
\hline 19 & $1.4 \mathrm{e}-13$ & $1.1 \mathrm{e}-01$ & $4.5 \mathrm{e}-01$ & 0.146 & $1.2 \mathrm{e}-14$ & $9.3 \mathrm{e}-02$ & $2.7 \mathrm{e}-01$ & 0.232 \\
\hline 20 & $6.8 \mathrm{e}-14$ & $1.8 \mathrm{e}-01$ & $7.0 \mathrm{e}-01$ & 0.202 & $2.4 \mathrm{e}-13$ & $1.2 \mathrm{e}-01$ & $7.1 \mathrm{e}-01$ & 0.282 \\
\hline 21 & $1.5 \mathrm{e}-13$ & $1.3 \mathrm{e}-01$ & $3.7 \mathrm{e}-01$ & 0.235 & $3.6 \mathrm{e}-14$ & $9.4 \mathrm{e}-02$ & $2.3 \mathrm{e}-01$ & 0.366 \\
\hline 22 & $1.8 \mathrm{e}-13$ & $2.3 \mathrm{e}-01$ & $5.5 \mathrm{e}-01$ & 0.291 & $7.0 \mathrm{e}-13$ & $1.4 \mathrm{e}-01$ & $5.1 \mathrm{e}-01$ & 0.469 \\
\hline 23 & $1.0 \mathrm{e}-12$ & $1.3 \mathrm{e}-01$ & $4.8 \mathrm{e}-01$ & 0.360 & $5.6 \mathrm{e}-13$ & $1.1 \mathrm{e}-01$ & $5.1 \mathrm{e}-01$ & 0.550 \\
\hline 24 & $1.1 \mathrm{e}-12$ & $2.1 \mathrm{e}-01$ & $4.8 \mathrm{e}-01$ & 0.389 & $9.9 \mathrm{e}-14$ & $1.9 \mathrm{e}-02$ & $4.2 \mathrm{e}-01$ & 0.610 \\
\hline 25 & $9.4 \mathrm{e}-13$ & $9.6 \mathrm{e}-02$ & $3.4 \mathrm{e}-01$ & 0.507 & $0.0 \mathrm{e}+00$ & $8.7 \mathrm{e}-02$ & $3.3 \mathrm{e}-01$ & 0.771 \\
\hline
\end{tabular}


TABLE 4

Stability Number

\begin{tabular}{|cc|ccc|ccc|}
\hline graph & & \multicolumn{3}{|c|}{ RSlocal } & \multicolumn{3}{c|}{ IDDM } \\
\hline name & $|V|$ & mean & $\max$ & cpu (s) & mean & $\max$ & cpu (s) \\
\hline theta10 & 500 & 47.0 & 50 & 0.686 & 47.0 & 51 & 0.620 \\
\hline theta12 & 600 & 49 & 50 & 0.949 & 49 & 54 & 0.872 \\
\hline theta42 & 200 & 15 & 17 & 0.254 & 15.5 & 18 & 0.265 \\
\hline G43 & 1000 & 180.5 & 188 & 0.671 & 189.0 & 195 & 0.497 \\
\hline G44 & 1000 & 182.0 & 190 & 0.654 & 191.0 & 199 & 0.503 \\
\hline G45 & 1000 & 179.0 & 188 & 0.667 & 187.5 & 197 & 0.495 \\
\hline G46 & 1000 & 180.0 & 186 & 0.651 & 189.0 & 196 & 0.505 \\
\hline G47 & 1000 & 184.0 & 190 & 0.689 & 191.5 & 200 & 0.487 \\
\hline G51 & 1000 & 332.0 & 336 & 0.813 & 343.0 & 346 & 0.603 \\
\hline G52 & 1000 & 330.0 & 335 & 0.837 & 341.0 & 344 & 0.616 \\
\hline G53 & 1000 & 330.0 & 334 & 0.783 & 340.0 & 343 & 0.557 \\
\hline G54 & 1000 & 323.0 & 330 & 0.725 & 334.0 & 339 & 0.532 \\
\hline sanr200-0.7 & 200 & 16.0 & 17 & 0.262 & 15.0 & 18 & 0.275 \\
\hline brock200-4 & 200 & 14.0 & 15 & 0.273 & 14.0 & 17 & 0.275 \\
\hline hamming-6-4 & 64 & 4.0 & 4 & 0.033 & 4.0 & 4 & 0.032 \\
\hline hamming-9-8 & 512 & 168.0 & 179 & 0.264 & 173.0 & 186 & 0.089 \\
\hline hamming-10-2 & 1024 & 65.0 & 67 & 0.911 & 66.0 & 70 & 0.844 \\
\hline hamming-11-2 & 2048 & 113.0 & 116 & 2.267 & 118.0 & 122 & 1.889 \\
\hline keller4 & 171 & 9.0 & 11 & 0.247 & 11.0 & 11 & 0.172 \\
\hline fap25 & 2118 & 78.0 & 80 & 29.471 & 79.0 & 82 & 25.063 \\
\hline 1dc.1024 & 1024 & 69.0 & 71 & 1.092 & 70.0 & 73 & 1.006 \\
\hline 1dc.2048 & 2048 & 119.0 & 123 & 2.679 & 125.0 & 129 & 2.290 \\
\hline 1et.512 & 512 & 91.0 & 96 & 0.202 & 92.0 & 96 & 0.175 \\
\hline 1et.1024 & 1024 & 154.0 & 158 & 0.426 & 159.0 & 162 & 0.363 \\
\hline 1et.2048 & 2048 & 270.0 & 275 & 0.971 & 289.0 & 296 & 0.894 \\
\hline 1tc.512 & 512 & 101.0 & 104 & 0.165 & 103.0 & 106 & 0.156 \\
\hline 1tc.1024 & 1024 & 174.0 & 180 & 0.357 & 183.0 & 187 & 0.302 \\
\hline 1tc.2048 & 2048 & 305.0 & 312 & 0.804 & 323.5 & 329 & 0.740 \\
\hline 1zc.512 & 512 & 51.0 & 54 & 0.299 & 51.5 & 55 & 0.286 \\
\hline 1zc.1024 & 1024 & 91.0 & 95 & 0.728 & 93.0 & 99 & 0.648 \\
\hline 1zc.2048 & 2048 & 160.0 & 164 & 1.740 & 169.0 & 175 & 1.388 \\
\hline 1zc.4096 & 4096 & 286.0 & 292 & 4.502 & 289.0 & 296 & 4.673 \\
\hline 2dc.512 & 512 & 10.0 & 10 & 2.303 & 10.0 & 11 & 2.001 \\
\hline
\end{tabular}


TABLE 5

The MSE of the eigenvector, RSlocal and IDDM for random dataset

\begin{tabular}{|c|c|c|c|c|c|c|c|c|c|}
\hline \multirow{2}{*}{$\mathrm{P}$} & \multicolumn{2}{|c|}{ eigs } & \multicolumn{3}{|c|}{ local } & \multicolumn{4}{|c|}{ IDDM } \\
\hline & mse & obj & mse1, obj1 & mse2, obj2 & $\mathrm{cpu}$ & mse1, obj1 & mse & obj2 & $\mathrm{cpu}$ \\
\hline \multicolumn{10}{|c|}{$\mathrm{N}=100$} \\
\hline 1.0 & $.45 \mathrm{e}-1$ & $3.10 \mathrm{e} 3$ & $1.19 \mathrm{e}-4 \quad 2.04 \mathrm{e} 2$ & \begin{tabular}{|ll}
$1.19 \mathrm{e}-4$ & $2.04 \mathrm{e} 2$
\end{tabular} & 0.7 & $1.19 \mathrm{e}-4 \quad 2.04 \mathrm{e} 2$ & $1.19 \mathrm{e}-2$ & $2.04 \mathrm{e} 2$ & 0.8 \\
\hline$\overline{9}$ & $3.31 \mathrm{e}-1$ & $3.28 \mathrm{e} 3$ & $9.98 \mathrm{e}-4 \quad 8.57 \mathrm{e} 2$ & $\begin{array}{|ll|}9.98 \mathrm{e}-4 & 8.57 \mathrm{e} 2 \\
\end{array}$ & 0.7 & 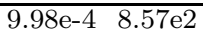 & $9.98 \mathrm{e}-2$ & $8.57 \mathrm{e} 2$ & 0.8 \\
\hline 8 & $3.77 \mathrm{e}-1$ & $3.56 \mathrm{e} 3$ & $\begin{array}{ll}7.01 \mathrm{e}-3 & 1.60 \mathrm{e} 3\end{array}$ & $\begin{array}{|ll|}7.01 \mathrm{e}-3 & 1.60 \mathrm{e} 3 \\
\end{array}$ & 0.7 & $\begin{array}{ll}7.01 \mathrm{e}-3 & 1.60 \mathrm{e} 3\end{array}$ & $7.01 \mathrm{e}-$ & $1.60 \mathrm{e} 3$ & 0.7 \\
\hline 0.7 & $3.97 \mathrm{e}-1$ & $3.88 \mathrm{e} 3$ & $\begin{array}{ll}3.86 \mathrm{e}-3 & 1.84 \mathrm{e} 3\end{array}$ & $\begin{array}{|ll|}3.86 \mathrm{e}-3 & 1.84 \mathrm{e} 3 \\
\end{array}$ & 0.7 & $\begin{array}{ll}3.86 \mathrm{e}-3 & 1.84 \mathrm{e} 3\end{array}$ & $3.86 \mathrm{e}-$ & $1.84 \mathrm{e} 3$ & 0.7 \\
\hline 0.6 & 2.18 & $4.84 \mathrm{e} 3$ & $3.17 \mathrm{e} 3$ & $3.17 \mathrm{e} 3$ & 0.5 & $\begin{array}{ll}9.78 \mathrm{e}-1 & 3.26 \mathrm{e} 3\end{array}$ & 1.25 & $3.17 \mathrm{e} 3$ & 0.6 \\
\hline 0.5 & $7.00 \mathrm{e}-1$ & $4.42 \mathrm{e} 3$ & $1.91 \mathrm{e}-1 \quad 3.29 \mathrm{e} 3$ & $\begin{array}{|ll|}1.91 \mathrm{e}-1 & 3.29 \mathrm{e} \\
\end{array}$ & 0.4 & $1.91 \mathrm{e}-1 \quad 3.29 \mathrm{e} 3$ & $1.91 \mathrm{e}-$ & $3.29 \mathrm{e} 3$ & 0.5 \\
\hline$\overline{0.4}$ & 2.89 & $5.15 \mathrm{e} 3$ & $4.21 \mathrm{e} 3$ & $4.21 \mathrm{e} 3$ & 0.5 & $4.10 \mathrm{e} 3$ & 1.67 & $4.10 \mathrm{e} 3$ & 0.6 \\
\hline 0.3 & 3.45 & $5.27 \mathrm{e} 3$ & $4.75 \mathrm{e} 3$ & $4.55 \mathrm{e} 3$ & 0.5 & $4.35 \mathrm{e} 3$ & 2.12 & $4.35 \mathrm{e} 3$ & 0.5 \\
\hline 0.2 & 3.56 & $5.37 \mathrm{e} 3$ & $4.84 \mathrm{e} 3$ & $4.84 \mathrm{e} 3$ & 0.5 & $4.69 \mathrm{e} 3$ & 2.28 & $4.69 \mathrm{e} 3$ & 0.5 \\
\hline 0.1 & 4.27 & $5.18 \mathrm{e} 3$ & $5.22 \mathrm{e} 3$ & $5.10 \mathrm{e} 3$ & 0.5 & $4.91 \mathrm{e} 3$ & 3.60 & $4.91 \mathrm{e} 3$ & 0.5 \\
\hline \multicolumn{10}{|c|}{$\mathrm{N}=500$} \\
\hline 1.0 & $3.42 \mathrm{e}-1$ & $7.76 \mathrm{e} 4$ & $1.33 \mathrm{e}-4 \quad 5.35 \mathrm{e} 3$ & \begin{tabular}{|ll}
$1.33 \mathrm{e}-4$ & $5.35 \mathrm{e} 3$
\end{tabular} & 9.8 & $1.33 \mathrm{e}-4 \quad 5.35 \mathrm{e} 3$ & $1.33 \mathrm{e}-2$ & $5.35 \mathrm{e} 3$ & 9.9 \\
\hline 0.9 & $3.42 \mathrm{e}-1$ & $8.39 \mathrm{e} 4$ & $4.98 \mathrm{e}-6 \quad 1.61 \mathrm{e} 4$ & $\begin{array}{|ll|}4.98 \mathrm{e}-6 & 1.61 \mathrm{e} \\
\end{array}$ & 9.9 & $4.98 \mathrm{e}-6 \quad 1.61 \mathrm{e} 4$ & $4.98 \mathrm{e}-\mathrm{t}$ & $1.61 \mathrm{e} 4$ & 8.5 \\
\hline 0.8 & $3.40 \mathrm{e}-1$ & $9.01 \mathrm{e} 4$ & $9.34 \mathrm{e}-4 \quad 3.55 \mathrm{e} 4$ & $\begin{array}{|cc|}9.34 \mathrm{e}-4 & 3.55 \mathrm{e} 4 \\
\end{array}$ & 6.4 & $9.34 \mathrm{e}-4 \quad 3.55 \mathrm{e} 4$ & $9.34 \mathrm{e}-2$ & $3.55 \mathrm{e} 4$ & 7.1 \\
\hline 0.7 & $3.35 \mathrm{e}-1$ & $9.60 \mathrm{e} 4$ & $\begin{array}{ll}.42 \mathrm{e}-5 & 4.51 \mathrm{e} 4\end{array}$ & $\begin{array}{|ll|}3.42 \mathrm{e}-5 & 4.51 \mathrm{e} 4 \\
\end{array}$ & 6.3 & $\begin{array}{cc}3.42 \mathrm{e}-5 & 4.51 \mathrm{e} 4\end{array}$ & $3.42 \mathrm{e}-\mathrm{s}$ & $4.51 \mathrm{e} 4$ & 7.4 \\
\hline 0.6 & $3.74 \mathrm{e}-1$ & $1.03 \mathrm{e} 5$ & $2.56 \mathrm{e}-3 \quad 6.31 \mathrm{e} 4$ & \begin{tabular}{|ll}
$2.56 \mathrm{e}-3$ & $6.31 \mathrm{e} 4$
\end{tabular} & 5.9 & $2.56 \mathrm{e}-3 \quad 6.31 \mathrm{e} 4$ & $2.56 \mathrm{e}-$ & $6.31 \mathrm{e} 4$ & 6.8 \\
\hline 0.5 & $3.74 \mathrm{e}-1$ & $1.10 \mathrm{e} 5$ & $6.28 \mathrm{e}-3 \quad 7.81 \mathrm{e} 4$ & 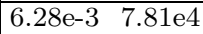 & 5.6 & $6.28 \mathrm{e}-3 \quad 7.81 \mathrm{e} 4$ & $6.28 \mathrm{e}-4$ & $7.81 \mathrm{e} 4$ & 5.9 \\
\hline$\overline{0.4}$ & $3.89 \mathrm{e}-1$ & $1.16 \mathrm{e} 5$ & 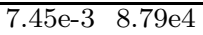 & $\begin{array}{|ll|}7.45 \mathrm{e}-3 & 8.79 \mathrm{e} \\
\end{array}$ & 5.0 & $\begin{array}{ll}7.45 \mathrm{e}-3 & 8.79 \mathrm{e} 4\end{array}$ & $7.45 \mathrm{e}-4$ & $8.79 \mathrm{e} 4$ & 5.5 \\
\hline 0.3 & $4.54 \mathrm{e}-1$ & $1.22 \mathrm{e} 5$ & $2.22 \mathrm{e}-2 \quad 1.03 \mathrm{e} 5$ & \begin{tabular}{|ll}
$2.22 \mathrm{e}-2$ & $1.03 \mathrm{e} 5$
\end{tabular} & 4.3 & $2.22 \mathrm{e}-2 \quad 1.03 \mathrm{e} 5$ & $2.22 \mathrm{e}-2$ & $1.03 \mathrm{e} 5$ & 5.2 \\
\hline$\overline{0.2}$ & $8.04 \mathrm{e}-1$ & $1.30 \mathrm{e} 5$ & $1.35 \mathrm{e}-1 \quad 1.16 \mathrm{e} 5$ & $1.35 \mathrm{e}-1 \quad 1.16 \mathrm{e} 5$ & 4.0 & $1.35 \mathrm{e}-1 \quad 1.16 \mathrm{e} 5$ & $1.35 \mathrm{e}-$ & $1.16 \mathrm{e} 5$ & 4.8 \\
\hline 0.1 & 4.03 & $1.38 \mathrm{e} 5$ & $1.33 \mathrm{e} 5$ & $1.28 \mathrm{e} 5$ & 4.3 & $1.26 \mathrm{e} 5$ & 2.59 & $1.26 \mathrm{e} 5$ & 4.8 \\
\hline \multicolumn{10}{|c|}{$\mathrm{N}=1000$} \\
\hline 1.0 & $34 \mathrm{e}-1$ & $3.08 \mathrm{e} 5$ & $1.43 \mathrm{e}-5 \quad 1.28 \mathrm{e} 4$ & $\begin{array}{|ll|}1.43 \mathrm{e}-5 & 1.28 \mathrm{e} 4 \\
\end{array}$ & 163 & $\begin{array}{ll}1.43 \mathrm{e}-5 & 1.28 \mathrm{e} 4\end{array}$ & $1.43 \mathrm{e}-$ & $1.28 \mathrm{e} 4$ & 87 \\
\hline 0.9 & $3.51 \mathrm{e}-1$ & $3.38 \mathrm{e} 5$ & 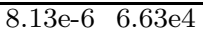 & $\begin{array}{|ll|}8.13 \mathrm{e}-6 & 6.63 \mathrm{e} 4 \\
\end{array}$ & 113 & $8.13 \mathrm{e}-6 \quad 6.63 \mathrm{e} 4$ & $8.13 \mathrm{e}-\mathrm{t}$ & $6.63 \mathrm{e} 4$ & 49 \\
\hline 0.8 & $3.51 \mathrm{e}-1$ & $3.63 \mathrm{e} 5$ & $\begin{array}{ll}9.46 \mathrm{e}-5 & 1.29 \mathrm{e} 5\end{array}$ & \begin{tabular}{|ll}
$9.46 \mathrm{e}-5$ & $1.29 \mathrm{e} 5$
\end{tabular} & 103 & $\begin{array}{ll}9.46 \mathrm{e}-5 & 1.29 \mathrm{e} 5\end{array}$ & $9.46 \mathrm{e}-!$ & $1.29 \mathrm{e} 5$ & 57 \\
\hline 0.7 & $3.54 \mathrm{e}-1$ & $3.89 \mathrm{e} 5$ & $3.20 \mathrm{e}-4 \quad 1.88 \mathrm{e} 5$ & \begin{tabular}{|ll}
$3.20 \mathrm{e}-4$ & $1.88 \mathrm{e} 5$ \\
\end{tabular} & 91 & $3.20 \mathrm{e}-4 \quad 1.88 \mathrm{e} 5$ & $3.20 \mathrm{e}-2$ & $1.88 \mathrm{e} 5$ & 42 \\
\hline 0.6 & $3.59 \mathrm{e}-1$ & $4.14 \mathrm{e} 5$ & $1.51 \mathrm{e}-4 \quad 2.36 \mathrm{e} 5$ & $1.51 \mathrm{e}-4 \quad 2.36 \mathrm{e} 5$ & 84 & $1.51 \mathrm{e}-4 \quad 2.36 \mathrm{e} 5$ & $1.51 \mathrm{e}-2$ & $2.36 \mathrm{e} 5$ & 33 \\
\hline 0.5 & $3.67 \mathrm{e}-1$ & $4.40 \mathrm{e} 5$ & $\begin{array}{ll}2.47 \mathrm{e}-5 & 2.84 \mathrm{e} 5\end{array}$ & $\begin{array}{|ll|}2.47 \mathrm{e}-5 & 2.84 \mathrm{e} 5 \\
\end{array}$ & 57 & $\begin{array}{ll}2.47 \mathrm{e}-5 & 2.84 \mathrm{e} 5\end{array}$ & $2.47 \mathrm{e}-$ & $2.84 \mathrm{e} 5$ & 35 \\
\hline 0.4 & $3.61 \mathrm{e}-1$ & $4.64 \mathrm{e} 5$ & $1.05 \mathrm{e}-3 \quad 3.43 \mathrm{e} 5$ & \begin{tabular}{|ll}
$1.05 \mathrm{e}-3$ & $3.43 \mathrm{e} 5$ \\
\end{tabular} & 50 & $\begin{array}{ll}1.05 \mathrm{e}-3 & 3.43 \mathrm{e} 5\end{array}$ & $1.05 \mathrm{e}-$ & $3.43 \mathrm{e} 5$ & 27 \\
\hline 0.3 & $3.97 \mathrm{e}-1$ & $4.90 \mathrm{e} 5$ & $1.31 \mathrm{e}-2 \quad 4.14 \mathrm{e} 5$ & $1.31 \mathrm{e}-2 \quad 4.14 \mathrm{e} 5$ & 34 & $1.31 \mathrm{e}-2 \quad 4.14 \mathrm{e} 5$ & $1.31 \mathrm{e}-$ & $4.14 \mathrm{e} 5$ & 23 \\
\hline 0.2 & $5.26 \mathrm{e}-1$ & $5.17 \mathrm{e} 5$ & $5.00 \mathrm{e}-2 \quad 4.65 \mathrm{e} 5$ & \begin{tabular}{|ll}
$5.00 \mathrm{e}-2$ & $4.65 \mathrm{e} 5$ \\
\end{tabular} & 18 & $5.00 \mathrm{e}-2 \quad 4.65 \mathrm{e} 5$ & $5.00 \mathrm{e}-$ & $4.65 \mathrm{e} 5$ & 18 \\
\hline 0.1 & 2.24 & $5.44 \mathrm{e} 5$ & $5.25 \mathrm{e} 5$ & $5.19 \mathrm{e} 5$ & 19 & $5.25 \mathrm{e} 5$ & 3.91 & $5.09 \mathrm{e} 5$ & 18 \\
\hline
\end{tabular}


TABLE 6

The MSE of the eigenvector, RSlocal and IDDM for dataset from [31]

\begin{tabular}{|c|c|c|c|c|c|c|c|c|}
\hline \multirow{2}{*}{ NSR } & \multicolumn{2}{|c|}{ eigs } & \multicolumn{3}{|c|}{$\overline{\text { RSlocal }}$} & \multicolumn{3}{|c|}{$\overline{\text { IDDM }}$} \\
\hline & mse & obj & mse1, obj1 & mse2, obj2 & $\mathrm{pu}$ & mse1, obj1 & $\mathrm{mse} 2, \mathrm{c}$ & cpu \\
\hline \multicolumn{9}{|c|}{$\mathrm{N}=100$} \\
\hline 1 & $0 \mathrm{e}-3$ & $5 \mathrm{e} 3$ & $4 \mathrm{e}-4 \quad 9.47 \mathrm{e} 2$ & $04 \mathrm{e}-4 \quad 9.47 \mathrm{e} 2$ & 0.5 & $99 \mathrm{e}-4 \quad 9.47 \mathrm{e} 2$ & $3.04 \mathrm{e}-4 \quad 9.47 \mathrm{e} 2$ & \\
\hline$\overline{2}$ & $1 \mathrm{e}-3$ & $0 \mathrm{e} 3$ & $04 \mathrm{e}-4 \quad 1.07 \mathrm{e} 3$ & $.04 \mathrm{e}-4 \quad 1.07 \mathrm{e} 3$ & $\overline{.5}$ & $4.95 \mathrm{e}-4 \quad 1.07 \mathrm{e} 3$ & $5.15 \mathrm{e}-4 \quad 1.07 \mathrm{e} 3$ & $\overline{.5}$ \\
\hline$\overline{4}$ & $6 \mathrm{e}-2$ & $2 \mathrm{e} 3$ & $60 \mathrm{e}-3 \quad 1.35 \mathrm{e} 3$ & $\begin{array}{|ll|}1.60 \mathrm{e}-3 & 1.35 \mathrm{e} 3 \\
\end{array}$ & 0.5 & $1.07 \mathrm{e}-3 \quad 1.35 \mathrm{e} 3$ & $1.10 \mathrm{e}-3 \quad 1.35 \mathrm{e} 3$ & 0.5 \\
\hline$\overline{8}$ & $8 \mathrm{e}-2$ & $01 \mathrm{e} 3$ & $19 \mathrm{e}-3 \quad 1.81 \mathrm{e} 3$ & $\begin{array}{|ll|}6.19 \mathrm{e}-3 & 1.81 \mathrm{e} 3 \\
\end{array}$ & 0.6 & $\begin{array}{|ll|}3.38 \mathrm{e}-3 & 1.79 \mathrm{e} \\
\end{array}$ & $3.38 \mathrm{e}-3 \quad 1.79 \mathrm{e} 3$ & 0.5 \\
\hline 16 & $1 \mathrm{e}-2$ & $69 \mathrm{e} 3$ & $.77 \mathrm{e}-2 \quad 2.50 \mathrm{e} 3$ & \begin{tabular}{|ll}
$3.77 \mathrm{e}-2$ & $2.50 \mathrm{e} 3$ \\
\end{tabular} & 0.6 & $3.04 \mathrm{e}-2 \quad 2.48 \mathrm{e} 3$ & $3.12 \mathrm{e}-2 \quad 2.48 \mathrm{e} 3$ & 0.5 \\
\hline$\overline{32}$ & $33 \mathrm{e}-1$ & $51 \mathrm{e} 3$ & $1.88 \mathrm{e}-1 \quad 3.34 \mathrm{e} 3$ & $\begin{array}{|ll|}1.88 \mathrm{e}-1 & 3.34 \mathrm{e} 3 \\
\end{array}$ & 0.5 & $1.88 \mathrm{e}-1 \quad 3.34 \mathrm{e} 3$ & $3.32 \mathrm{e} 3$ & 0.4 \\
\hline 34 & 0 & $4.32 \mathrm{e} 3$ & $4.03 \mathrm{e} 3$ & $4.03 \mathrm{e} 3$ & 0.5 & $4.03 \mathrm{e} 3$ & $3.89 \mathrm{e} 3$ & $\overline{0.4}$ \\
\hline 128 & 3.28 & $4.79 \mathrm{e} 3$ & $4.64 \mathrm{e} 3$ & $4.64 \mathrm{e} 3$ & 0.4 & $4.64 \mathrm{e} 3$ & $4.45 \mathrm{e} 3$ & 0.4 \\
\hline 256 & 4.10 & $5.04 \mathrm{e} 3$ & $4.99 \mathrm{e} 3$ & $4.99 \mathrm{e} 3$ & 0.4 & $4.99 \mathrm{e} 3$ & $4.69 \mathrm{e} 3$ & 0.4 \\
\hline 512 & 4.97 & $5.15 \mathrm{e} 3$ & $5.09 \mathrm{e} 3$ & $5.09 \mathrm{e} 3$ & 0.4 & $5.03 \mathrm{e} 3$ & $4.93 \mathrm{e} 3$ & 0.4 \\
\hline \multicolumn{9}{|c|}{$\mathrm{N}=500$} \\
\hline 1 & $20 \mathrm{e}-3$ & $2.53 \mathrm{e} 4$ & $54 \mathrm{e}-4$ & \begin{tabular}{|ll}
$1.54 \mathrm{e}-4$ & $2.43 \mathrm{e} 4$
\end{tabular} & 16 & $\begin{array}{|ll|}1.54 \mathrm{e}-4 & 2.43 \mathrm{e} \\
\end{array}$ & $\begin{array}{|ll|}1.54 \mathrm{e}-4 & 2.43 \mathrm{e} 4 \\
\end{array}$ & 7.8 \\
\hline 2 & $77 \mathrm{e}-3$ & $2.87 \mathrm{e} 4$ & $2.94 \mathrm{e}-4 \quad 2.75 \mathrm{e} 4$ & $2.94 \mathrm{e}-4 \quad 2.75 \mathrm{e} 4$ & 17 & $2.94 \mathrm{e}-4 \quad 2.75 \mathrm{e} 4$ & $2.94 \mathrm{e}-4 \quad 2.75 \mathrm{e} 4$ & 11 \\
\hline 4 & $5.10 \mathrm{e}-3$ & $3.60 \mathrm{e} 4$ & $\begin{array}{|ll|}7.58 \mathrm{e}-4 & 3.39 \mathrm{e} 4 \\
\end{array}$ & $\begin{array}{|ll|}7.58 \mathrm{e}-4 & 3.39 \mathrm{e} 4 \\
\end{array}$ & 16 & $7.07 \mathrm{e}-4 \quad 3.39 \mathrm{e} 4$ & $\begin{array}{|ll|}7.07 \mathrm{e}-4 & 3.39 \mathrm{e} 4 \\
\end{array}$ & \begin{tabular}{|l|}
7.1 \\
\end{tabular} \\
\hline 8 & $91 \mathrm{e}-2$ & $4.86 \mathrm{e} 4$ & \begin{tabular}{|ll}
$3.14 \mathrm{e}-3$ & $4.53 \mathrm{e} 4$ \\
\end{tabular} & $\begin{array}{|ll|}3.14 \mathrm{e}-3 & 4.53 \mathrm{e} 4 \\
\end{array}$ & 17 & \begin{tabular}{|ll}
$.14 \mathrm{e}-3$ & $4.53 \mathrm{e} 4$ \\
\end{tabular} & \begin{tabular}{|ll}
$3.14 \mathrm{e}-3$ & $4.53 \mathrm{e} 4$ \\
\end{tabular} & 7.1 \\
\hline 16 & $35 \mathrm{e}-2$ & $.58 \mathrm{e} 4$ & $\begin{array}{|ll|}1.81 \mathrm{e}-2 & 6.19 \mathrm{e} 4 \\
\end{array}$ & \begin{tabular}{|ll}
$1.81 \mathrm{e}-2$ & $6.19 \mathrm{e} 4$ \\
\end{tabular} & 8.7 & $\begin{array}{|ll|}1.81 \mathrm{e}-2 & 6.19 \mathrm{e} 4 \\
\end{array}$ & $\begin{array}{|ll|}1.81 \mathrm{e}-2 & 6.19 \mathrm{e} 4 \\
\end{array}$ & 7.6 \\
\hline 32 & $2.18 \mathrm{e}-1$ & $62 \mathrm{e} 4$ & $1.34 \mathrm{e}-1 \quad 8.25 \mathrm{e} 4$ & $1.34 \mathrm{e}-1 \quad 8.25 \mathrm{e} 4$ & 5.5 & $1.34 \mathrm{e}-1 \quad 8.25 \mathrm{e} 4$ & $1.34 \mathrm{e}-1$ & 6.8 \\
\hline 64 & 75 & $7 \mathrm{e} 5$ & $9.86 \mathrm{e} 4$ & $9.86 \mathrm{e} 4$ & 5.6 & $9.86 \mathrm{e} 4$ & $9.52 \mathrm{e} 4$ & 9.3 \\
\hline 128 & 52 & $1.21 \mathrm{e} 5$ & $1.14 \mathrm{e} 5$ & $1.14 \mathrm{e} 5$ & 5.7 & $1.14 \mathrm{e} 5$ & $1.11 \mathrm{e} 5$ & 8.8 \\
\hline$\overline{256}$ & 3.49 & $29 \mathrm{e} 5$ & $1.24 \mathrm{e} 5$ & $1.24 \mathrm{e} 5$ & 5.0 & $1.24 \mathrm{e} 5$ & $1.22 \mathrm{e} 5$ & 5.5 \\
\hline 12 & 59 & $32 \mathrm{e} 5$ & $1.32 \mathrm{e} 5$ & $1.27 \mathrm{e} 5$ & 6.1 & $1.26 \mathrm{e} 5$ & $1.26 \mathrm{e} 5$ & 5.9 \\
\hline \multicolumn{9}{|c|}{$\mathrm{N}=1000$} \\
\hline 1 & $7 \mathrm{e}-4$ & $0 \mathrm{e} 5$ & $\begin{array}{ll}25 \mathrm{e}-4 & 9.73 \mathrm{e} 4\end{array}$ & $1.25 \mathrm{e}-4 \quad 9.73 \mathrm{e} 4$ & 59 & $1.25 \mathrm{e}-4 \quad 9.73 \mathrm{e} 4$ & $1.25 \mathrm{e}-4 \quad 9.73 \mathrm{e} 4$ & 51 \\
\hline 2 & $6 \mathrm{e}-3$ & $5 \mathrm{e} 5$ & $\begin{array}{|ll|}2.58 \mathrm{e}-4 & 1.10 \mathrm{e} 5 \\
\end{array}$ & $\begin{array}{|ll|}2.58 \mathrm{e}-4 & 1.10 \mathrm{e} 5 \\
\end{array}$ & 75 & $2.58 \mathrm{e}-4 \quad 1.10 \mathrm{e} 5$ & $\begin{array}{|ll|}2.58 \mathrm{e}-4 & 1.10 \mathrm{e} 5 \\
\end{array}$ & 67 \\
\hline 4 & $6 e-3$ & $1.44 \mathrm{e} 5$ & $6.60 \mathrm{e}-4 \quad 1.36 \mathrm{e} 5$ & $6.60 \mathrm{e}-4 \quad 1.36 \mathrm{e} 5$ & 65 & $6.60 \mathrm{e}-4 \quad 1.36 \mathrm{e} 5$ & $\begin{array}{|ll|}6.60 \mathrm{e}-4 & 1.36 \mathrm{e} 5 \\
\end{array}$ & 58 \\
\hline 8 & $1.81 \mathrm{e}-2$ & $1.94 \mathrm{e} 5$ & \begin{tabular}{|ll}
$2.46 \mathrm{e}-3$ & $1.81 \mathrm{e} 5$ \\
\end{tabular} & \begin{tabular}{|ll}
$2.46 \mathrm{e}-3$ & $1.81 \mathrm{e} 5$
\end{tabular} & 60 & $\begin{array}{|ll|}2.46 \mathrm{e}-3 & 1.81 \mathrm{e} 5 \\
\end{array}$ & $\begin{array}{|ll|}2.46 \mathrm{e}-3 & 1.81 \mathrm{e} 5 \\
\end{array}$ & 45 \\
\hline 16 & $6.41 \mathrm{e}-2$ & $2.63 \mathrm{e} 5$ & $1.43 \mathrm{e}-2 \quad 2.47 \mathrm{e} 5$ & $1.43 \mathrm{e}-2 \quad 2.47 \mathrm{e} 5$ & 43 & $1.43 \mathrm{e}-2 \quad 2.47 \mathrm{e} 5$ & $1.43 \mathrm{e}-2 \quad 2.47 \mathrm{e} 5$ & 55 \\
\hline 32 & $2.32 \mathrm{e}-1$ & $3.44 \mathrm{e} 5$ & $1.43 \mathrm{e}-1 \quad 3.30 \mathrm{e} 5$ & $1.43 \mathrm{e}-1 \quad 3.30 \mathrm{e} 5$ & 28 & $1.43 \mathrm{e}-1 \quad 3.30 \mathrm{e} 5$ & $3.23 \mathrm{e} 5$ & 35 \\
\hline 64 & 1.78 & $4.29 \mathrm{e} 5$ & $3.93 \mathrm{e} 5$ & $3.93 \mathrm{e} 5$ & 32 & $3.93 \mathrm{e} 5$ & $3.82 \mathrm{e} 5$ & 37 \\
\hline 128 & 2.50 & $4.83 \mathrm{e} 5$ & $4.52 \mathrm{e} 5$ & $4.52 \mathrm{e} 5$ & 36 & $4.52 \mathrm{e} 5$ & $4.44 \mathrm{e} 5$ & 30 \\
\hline 256 & 3.48 & $5.17 \mathrm{e} 5$ & $4.98 \mathrm{e} 5$ & $4.98 \mathrm{e} 5$ & 33 & $4.98 \mathrm{e} 5$ & $4.89 \mathrm{e} 5$ & 37 \\
\hline 512 & 4.62 & $5.32 \mathrm{e} 5$ & $5.32 \mathrm{e} 5$ & $5.08 \mathrm{e} 5$ & 30 & $5.32 \mathrm{e} 5$ & $5.05 \mathrm{e} 5$ & 29 \\
\hline
\end{tabular}




\section{REFERENCES}

[1] P. A. Absil, R. Mahony, and R. Sepulchre, Optimization algorithms on matrix manifolds, Princeton University Press, Princeton, NJ, 2008, doi:10.1515/9781400830244.

[2] M. Aharon, M. Elad, and A. Bruckstein, rmk-svd: An algorithm for designing overcomplete dictionaries for sparse representation, IEEE Transactions on signal processing, 54 (2006), pp. 4311-4322.

[3] F. Aluffi-Pentini, V. Parisi, and F. Zirilli, Global optimization and stochastic differential equations, Journal of optimization theory and applications, 47 (1985), pp. 1-16, doi:10.1007/BF00941312.

[4] P. T. Boufounos and R. G. Baraniuk, 1-bit compressive sensing, in Information Sciences and Systems, 2008. CISS 2008. 42nd Annual Conference on, IEEE, 2008, pp. 16-21.

[5] J.-F. Cai, H. Ji, Z. Shen, And G.-B. Ye, Data-driven tight frame construction and image denoising, Applied and Computational Harmonic Analysis, 37 (2014), pp. 89-105.

[6] T.-S. Chiang, C.-R. Hwang, and S. J. Sheu, Diffusion for Global Optimization in $\mathbb{R}^{n}$, SIAM Journal on Control and Optimization, 25 (1987), pp. 737-753, doi:10.1137/0325042.

[7] S.-N. CHOW, T.-S. YANG, AND H.-M. ZHOU, GLOBAL OPTIMIZATIONS BY INTERMITTENT DIFFUSION, in Chaos, CNN, Memristors and Beyond, WORLD SCIENTIFIC, Feb. 2013, pp. 466-479, doi:10.1142/9789814434805_0037.

[8] A. Edelman, T. A. Arias, And S. T. Smith, The Geometry of Algorithms with Orthogonality Constraint, SIAM Journal on Matrix Analysis and Applications, 20 (1998), pp. 303-353, doi:10.1137/S0895479895290954.

[9] S. Geman And C.-R. Hwang, Diffusions for Global Optimization, SIAM Journal on Control and Optimization, 24 (1986), pp. 1031-1043, doi:10.1137/0324060.

[10] B. GIDAs, Global optimization via the Langevin equation, in 1985 24th IEEE Conference on Decision and Control, IEEE, 1985, pp. 774-778, doi:10.1109/CDC.1985.268602.

[11] D. Goldfarb, Z. Wen, And W. Yin, A curvilinear search method for p-harmonic flows on spheres, SIAM Journal on Imaging Sciences, 2 (2009), pp. 84-109.

[12] X. GU AND S.-T. YAU, Global conformal surface parameterization, in Proceedings of the 2003 Eurographics/ACM SIGGRAPH symposium on Geometry processing, Eurographics Association, 2003, pp. 127-137.

[13] O. Henkel, Sphere-Packing Bounds in the Grassmann and Stiefel Manifolds, Institute of Electrical and Electronics Engineers. Transactions on Information Theory, 51 (2005), pp. 34453456, doi:10.1109/TIT.2005.855594.

[14] E. Hsu, Stochastic Analysis on Manifolds, vol. 38 of Graduate Studies in Mathematics, American Mathematical Society, Providence, Rhode Island, Feb. 2002, doi:10.1090/gsm/038.

[15] R. Lai, Z. Wen, W. Yin, X. Gu, AND L. M. Lui, Folding-free global conformal mapping for genus-0 surfaces by harmonic energy minimization, Journal of Scientific Computing, 58 (2014), pp. 705-725.

[16] J. N. Laska, Z. Wen, W. Yin, And R. G. Baraniuk, Trust, but verify: Fast and accurate signal recovery from 1-bit compressive measurements, IEEE Transactions on Signal Processing, 59 (2011), pp. 5289-5301.

[17] S.-Y. Lin AND M. Luskin, Relaxation methods for liquid crystal problems, SIAM Journal on Numerical Analysis, 26 (1989), pp. 1310-1324.

[18] C. Ling, J. NiE, L. QI, AND Y. YE, Biquadratic optimization over unit spheres and semidefinite programming relaxations, SIAM J. Optim., 20 (2009), pp. 1286-1310, doi:10.1137/080729104.

[19] X. Liu, Z. Wen, X. Wang, M. Ulbrich, and Y. Yuan, On the analysis of the discretized Kohn-Sham density functional theory, SIAM J. Numer. Anal., 53 (2015), pp. 1758-1785, doi:10.1137/140957962.

[20] D. MARINGer AND P. PARPAS, Global optimization of higher order moments in portfolio selection, Journal of Global Optimization, 43 (2007), pp. 219-230, doi:10.1007/s10898-007-9224-3.

[21] P. A. Markowich and C. Villani, On the trend to equilibrium for the Fokker-Planck equation: an interplay between physics and functional analysis, Matemática Contemporânea, 19 (2000), pp. 1-29.

[22] T. S. Motzkin and E. G. Straus, Maxima for graphs and a new proof of a theorem of Turán, Canadian Journal of Mathematics. Journal Canadien de Mathématiques, 17 (1965), pp. 533-540, doi:10.4153/CJM-1965-053-6.

[23] J. Nocedal And S. J. Wright, Numerical Optimization, Springer Series in Operations Research and Financial Engineering, Springer, New York, second ed., 2006.

[24] B. Ø KSEndal, Stochastic differential equations, Universitext, Springer-Verlag, Berlin, Berlin, 
Heidelberg, sixth ed., 2003, doi:10.1007/978-3-642-14394-6.

[25] J. Oprea, Differential geometry and its applications, Classroom Resource Materials Series, Mathematical Association of America, Washington, DC, second ed., 2007.

[26] V. Ozolinšs, R. Lai, R. CAFLisch, And S. Osher, Compressed modes for variational problems in mathematics and physics, Proceedings of the National Academy of Sciences, 110 (2013), pp. $18368-18373$.

[27] P. Parpas and B. Rustem, An Algorithm for the Global Optimization of a Class of Continuous Minimax Problems, Journal of optimization theory and applications, 141 (2008), pp. 461473, doi:10.1007/s10957-008-9473-4.

[28] P. Parpas AND B. Rustem, Convergence analysis of a global optimization algorithm using stochastic differential equations, Journal of Global Optimization, 45 (2009), pp. 95-110, doi:10.1007/s10898-008-9397-4.

[29] P. Parpas, B. Rustem, and E. N. Pistikopoulos, Linearly Constrained Global Optimization and Stochastic Differential Equations, Journal of Global Optimization, 36 (2006), pp. 191217, doi:10.1007/s10898-006-9026-z.

[30] P. Parpas, B. Rustem, And E. N. Pistikopoulos, Global optimization of robust chance constrained problems, Journal of Global Optimization, 43 (2007), pp. 231-247, doi:10.1007/s10898-007-9244-z.

[31] A. Singer and Y. Shkolnisky, Three-Dimensional Structure Determination from Common Lines in Cryo-EM by Eigenvectors and Semidefinite Programming, SIAM Journal on Imaging Sciences, 4 (2011), pp. 543-572, doi:10.1137/090767777.

[32] B. Tang, G. Sapiro, and V. Caselles, Color image enhancement via chromaticity diffusion, IEEE Transactions on Image Processing, 10 (2001), pp. 701-707.

[33] L. A. Vese AND S. J. Osher, Numerical methods for p-harmonic flows and applications to image processing, SIAM Journal on Numerical Analysis, 40 (2002), pp. 2085-2104.

[34] C. Villani, Optimal Transport, vol. 338 of Grundlehren der mathematischen Wissenschaften, Springer Berlin Heidelberg, Berlin, Heidelberg, 2009, doi:10.1007/978-3-540-71050-9.

[35] Z. Wen, A. Milzarek, M. Ulbrich, and H. Zhang, Adaptive regularized self-consistent field iteration with exact Hessian for electronic structure calculation, SIAM J. Sci. Comput., 35 (2013), pp. A1299-A1324, doi:10.1137/120894385.

[36] Z. Wen AND W. Yin, A feasible method for optimization with orthogonality constraints, Mathematical programming, 142 (2012), pp. 397-434, doi:10.1007/s10107-012-0584-1.

[37] G. Yin AND K. Yin, Global Optimization Using Diffusion Perturbations with Large Noise Intensity, Acta Mathematicae Applicatae Sinica, English Series, 22 (2006), pp. 529-542, doi:10.1007/s10255-006-0328-1. 